\title{
Detection of highly-ionized diffuse gas in the Galactic plane ${ }^{\star}$
}

\author{
M. Mizutani ${ }^{1, \star \star}$, T. Onaka ${ }^{1}$, and H. Shibai ${ }^{2}$ \\ 1 Department of Astronomy, Graduate School of Science, University of Tokyo, Tokyo 113-0033, Japan \\ 2 Department of Physics, Graduate School of Science, Nagoya University, Nagoya 464-8602, Japan
}

Received 1 September 2000 / Accepted 5 November 2001

\begin{abstract}
We obtained maps of the central $40^{\prime} \times 20^{\prime}$ region of the Carina nebula, an active star forming region in the southern Milky Way, in various emission lines in the spectral range 43-197 $\mu \mathrm{m}$ with the LWS on board the ISO. This paper reports on the results of [O III] 52, $88 \mu \mathrm{m}$, [N III] $57 \mu \mathrm{m}$, and [N II] $122 \mu \mathrm{m}$ lines. These ionized lines have been detected in the entire observed area, not only in the optically bright $\mathrm{H}$ II region, but also in the molecular cloud direction and the region surrounding the Carina nebula. The electron density was derived from the [O III] lines and two distinct components were seen in the electron density map. One is a component with a density of $100-350 \mathrm{~cm}^{-3}$, which encloses two H II regions, Car I and Car II. The other is an extended diffuse component, in which the ratio of the [O III] lines is nearly at the low electron density limit $\left(n_{\mathrm{e}}<100 \mathrm{~cm}^{-3}\right)$. The observed $[\mathrm{N} \mathrm{III}] /[\mathrm{O} \mathrm{III}]$ line intensity ratio also supports low electron density of the extended gas component. The diffuse component was detected in the entire observed region, which corresponds to $\sim 30 \mathrm{pc}$ at the distance of the Carina nebula. The present LWS observations of the ionic lines indicate the existence of highly-ionized diffuse gas of low electron density extending around the Carina nebula.
\end{abstract}

Key words. infrared: ISM: lines and bands - H II regions - ISM: individual objects: Carina nebula

\section{Introduction}

The Carina nebula is one of the most active regions on the Galactic plane. It covers an area of about $4^{\circ} \times 4^{\circ}$ in visual wavelengths. The brightest region of the nebula has a triangular shape with two bright $\mathrm{H}$ II regions, known as Car I and II. This region has several star clusters consisting of early-type stars and Wolf-Rayet stars (for reviews, see Walborn 1995 and Feinstein 1995). The most influential clusters are two OB star clusters, Trumpler (Tr) 14 and 16. These clusters contain numerous early O-type stars. Tr 14 is a compact star cluster situated adjacent to the western dust lane and $\operatorname{Tr} 16$ is an open cluster centered at the vertex of the dust lane. An IR bright star $\eta$ Car is a member of $\operatorname{Tr} 16$. Tr 14 and $\operatorname{Tr} 16$ are major heating sources in this region. According to the picture commonly accepted, Car I is excited by the star cluster Tr 14 and Car II by Tr 16 (Harvey et al. 1979).

Send offprint requests to: T. Onaka,

e-mail: onaka@astron.s.u-tokyo.ac.jp

* Based on observations with ISO, an ESA project with instruments funded by ESA Member States (especially the PI countries: France, Germany, The Netherlands and the UK) and with the participation of ISAS and NASA.

$\star \star$ Present address: Central Research Laboratory, Hitachi, Ltd., 1-280 Higashi-Koigakubo, Kokubunji, Tokyo 185-8601, Japan.
The Carina nebula has been observed in various wavelengths and the spatial structure and kinematics of the nebular cloud have been studied extensively. In the radio continuum, there are two strong thermal emission peaks, Car I and Car II (Gardner \& Morimoto 1968) lying within a widespread thermal component (Gardner et al. 1970; Retallack 1983). High-resolution radio observations show the filamentary nature of the surrounding emission (Whiteoak 1994). Molecular gas is concentrated in the dark lane which causes optical obscuration. CO observations indicate the presence of massive molecular clouds (Grabelsky et al. 1988) and the complex clumpy morphology of the molecular gas in the Carina region (de Graauw et al. 1981; Whiteoak \& Otrupcek 1984; Cox \& Bronfman 1995; Brooks et al. 1998). Far-infrared emission associated with the dust lane has also been observed (Harvey et al. 1979; Ghosh et al. 1988). The velocity structure is explained by a model of molecular gas surrounding the ionized gas and expanding at about $7 \mathrm{~km} \mathrm{~s}^{-1}$ from a point $15^{\prime}$ southwest of $\eta$ Car (Dickel 1974). Optical observations show strong line splitting near Car II, suggesting that the ionized gas is expanding at $25 \mathrm{~km} \mathrm{~s}^{-1}$ (Deharveng \& Maucherat 1975). The Carina complex is one of the most fascinating and puzzling objects in the Galaxy and it is suitable for the study of the physical conditions of diffuse interstellar medium associated with OB clusters. The distance to the Carina nebula is estimated as about 2.7 kpc (Grabelsky et al. 1988). 


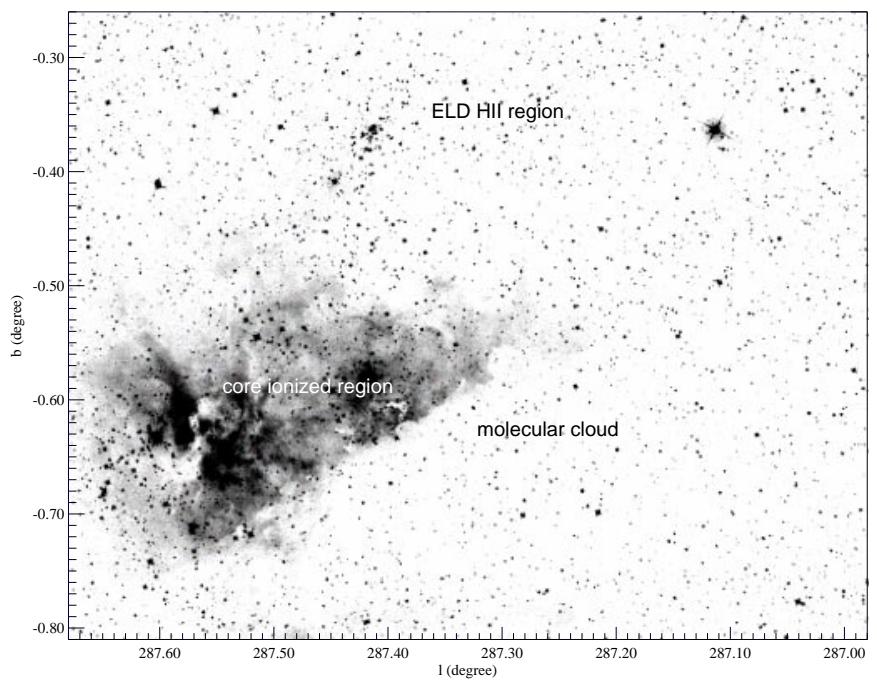

Fig. 1. The visual image of the observed region from the Digital Sky Survey. Locations of three distinct regions are indicated (see text).

The Long Wavelength Spectrometer (LWS; Clegg et al. 1996) on board the Infrared Space Observatory (ISO; Kessler et al. 1996) offers a unique capability of measuring the spectrum in the far-infrared wavelength range. The bulk of energy emitted by interstellar dust has its peak in the far-infrared and a number of spectral lines from the gaseous species of interstellar medium (ISM) are also seen in this spectral range. Spectral observations in the far-infrared wavelengths will thus provide important information in the study of the diffuse ISM.

In the guaranteed time observation TONAKA. WDISM1, we have made mapping observations with the LWS in order to investigate the nature of diffuse ISM of this region. In this paper we report on the results of two [O III] lines $(52,88 \mu \mathrm{m})$, one [N III] line $(57 \mu \mathrm{m})$, and one $[\mathrm{N} \mathrm{II}]$ line $(122 \mu \mathrm{m})$, and discuss the properties of the ionized gas emitting these lines.

\section{Observations and data reduction}

The observed region is a rectangular area of the central part of the Carina region of $l=287^{\circ} .0-287^{\circ} .65$ and $b=-0.78--0.23$. It covers approximately an area of $40^{\prime} \times 20^{\prime}$, which encloses two well-known H II regions, Car I and Car II, and a famous IR bright star, $\eta$ Carina. Figure 1 shows the visual image of the observed region from the Digital Sky Survey ${ }^{1}$. In the eastern part of the observed region $\left(l>287^{\circ} .3\right)$, the $\mathrm{H}$ II regions are located in front

1 Based on photographic data of the National Geographic Society - Palomar Observatory Sky Survey (NGS-POSS) obtained using the Oschin Telescope on Palomar Mountain. The NGS-POSS was funded by a grant from the National Geographic Society to the California Institute of Technology. The plates were processed into the present compressed digital form with their permission. The Digitized Sky Survey was produced at the Space Telescope Science Institute under US Government grant NAG W-2166. of molecular clouds, while in the western part the nebula is behind the molecular clouds. CO $(J=2-1)$ line observations suggest the spatial structure of an extended molecular cloud wrapped partly around bright $\mathrm{H}$ II regions (de Graauw et al. 1981).

The observations were made with the LWS on board the ISO in the full grating scan mode (LWS01: 43$197 \mu \mathrm{m})$. The LWS was operated in the raster scan mode with a step of $180^{\prime \prime}$ and 6 raster observations were executed to cover the target 132 positions. The spectrum at each position was obtained by averaging over 4 individual spectral scans and the total exposure time for each grating position was $0.5 \mathrm{~s}$. The spectral resolution was approximately 200 and the beam size was approximately 80" (Burgdorf et al. 1997; Gry 2000). Thus the present observations provide an undersampling map.

In addition to the main mapping observation of the Carina nebular region, we also made observations in the same observing mode at 4 outer positions; $(l, b)=$ $\left(287^{\circ} .306,+0.498\right),\left(287^{\circ} 356,+0^{\circ} .498\right),\left(287^{\circ} 306,+0.831\right)$, and $\left(287^{\circ} 356,+0.831\right)$. These data were reduced in a similar manner.

We have used the pipeline OLP.8 version products for the present study. The spectra were defringed by our own software, which was developed based on the ISO Spectral Analysis Package (ISAP ${ }^{2}$ ) subroutines. After rebinning and averaging over 4 scans, the line intensities were derived from the Gaussian fit of the line profile by the leastsquares method, assuming that the baseline was linear. An upper limit of the undetected line intensity was estimated from the Gaussian of the instrumental spectral width with a peak height of the statistical error calculated in the averaging process of each scan.

The accuracy of absolute calibration is estimated as $30 \%$ (Gry 2000). The calibration of the LWS is based on the point source observations and therefore the intensities of extended sources have to be corrected for the beam size effect. We adopted the beam sizes and the extended source correction factors provided by Gry (2000).

\section{Results}

\subsection{Intensity distributions}

In the observed spectra several ionic and atomic finestructure lines, such as [O III] $52,88 \mu \mathrm{m},[\mathrm{O}$ I $] 63,145 \mu \mathrm{m}$, [N III] $57 \mu \mathrm{m},[\mathrm{N} \mathrm{II}] 122 \mu \mathrm{m}$, and [C II] $158 \mu \mathrm{m}$ lines are clearly detected at almost all the observed positions. In this paper we report the results of the lines of [O III] 52, $88 \mu \mathrm{m},[\mathrm{N} \mathrm{III}] 57 \mu \mathrm{m}$, and [N II] $122 \mu \mathrm{m}$. The intensities for each observed position of the main mapping region are given in Table 1 , where the intensities largerthan $2 \sigma$ are

\footnotetext{
${ }^{2}$ The ISO Spectral Analysis Package (ISAP) is a joint development by the LWS and SWS Instrument Teams and Data Centers. Contributing institutes are CESR, IAS, IPAC, MPE, RAL and SRON.
} 

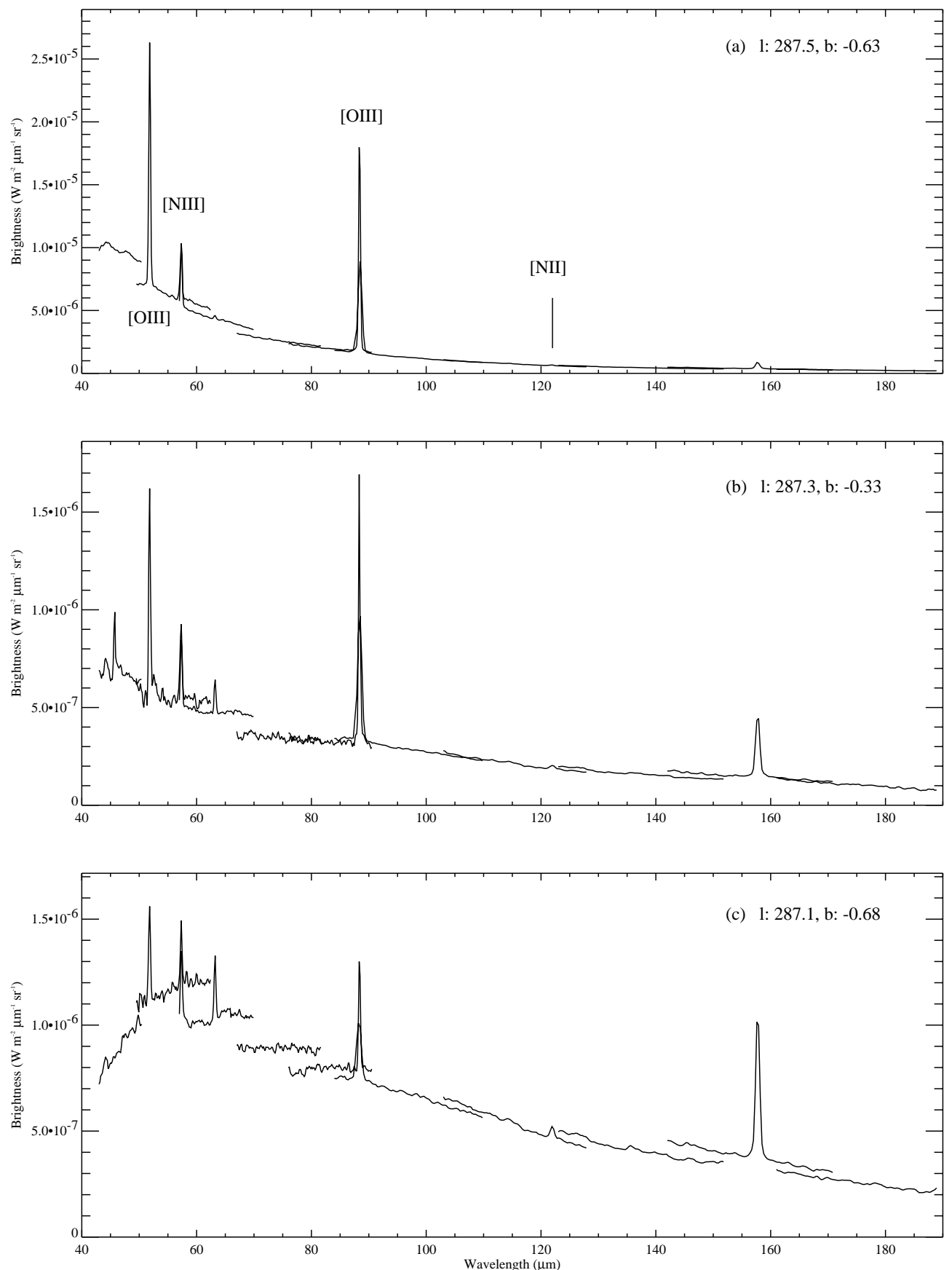

Fig. 2. Sample spectra of the LWS observations. The top figure a) shows a spectrum of the bright ionized region and the middle b) is a spectrum of the surrounding region. The bottom c) shows a spectrum of the molecular cloud region.

listed with the errors in the parentheses. The errors come mostly from the uncertainty in the estimate of the baseline and do not include the uncertainty in the absolute calibration of $30 \%$. Relative uncertainties among the detector channels can be estimated from the gaps seen in the continuum flux (see next subsection). For the [O III] $88 \mu \mathrm{m}$ line, we use the SW5 channel data rather than the LW1 data because the SW5 data have a higher spectral resolution and the Gaussian fit can be made with better accuracy.
Based on the observed spectra, we classify the observed area into three different regions: the highly ionized optically bright region $\left(l>287^{\circ} 3, b<-0\right.$. 4$)$, the region surrounding the Carina Nebula $(b>-0.4)$, and the molecular cloud region extended in the western side of the observed area $\left(l<287^{\circ} .3, b<-0.4\right)$ (see Fig. 1$)$. Typical spectra of the three regions are shown in Fig. 2. The spectrum of the optically bright region shows strong fine-structure lines, [O III] 52, $88 \mu \mathrm{m}$ and [N III] $57 \mu \mathrm{m}$ (Fig. 2a). The spectrum of the surrounding region has 
(a) $[\mathrm{OIII}] 52 \mu \mathrm{m}$

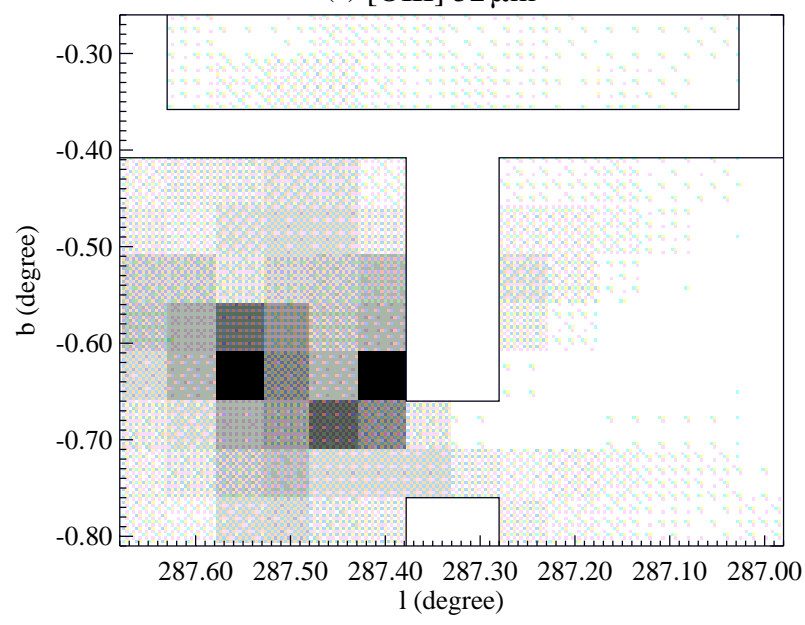

$\left[\mathrm{W} \mathrm{m}^{-2} \mathrm{str}^{-1}\right]$

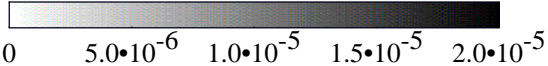

(c) $[\mathrm{NIII}] 57 \mu \mathrm{m}$

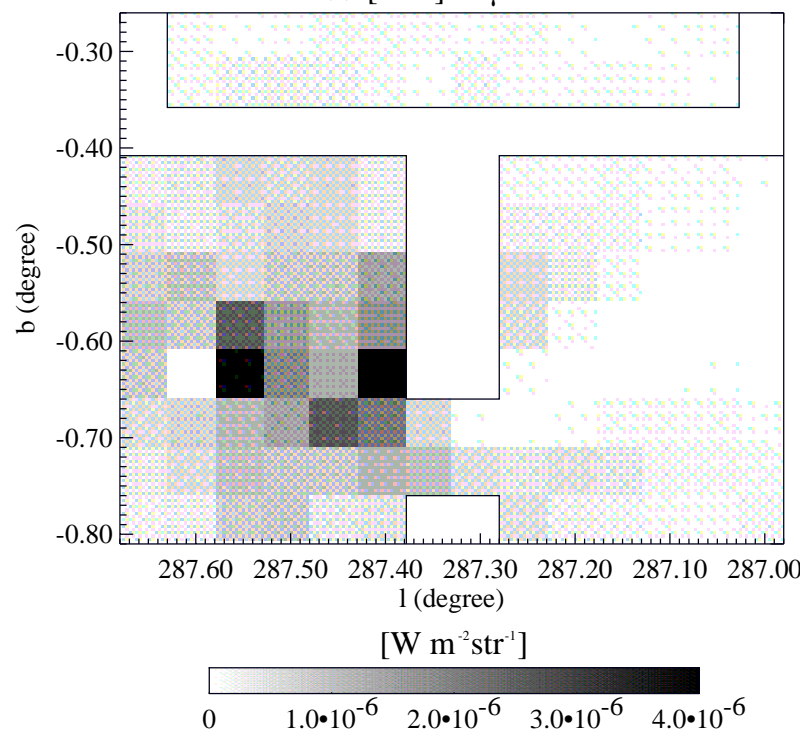

(b) $[\mathrm{OIII}] 88 \mu \mathrm{m}$

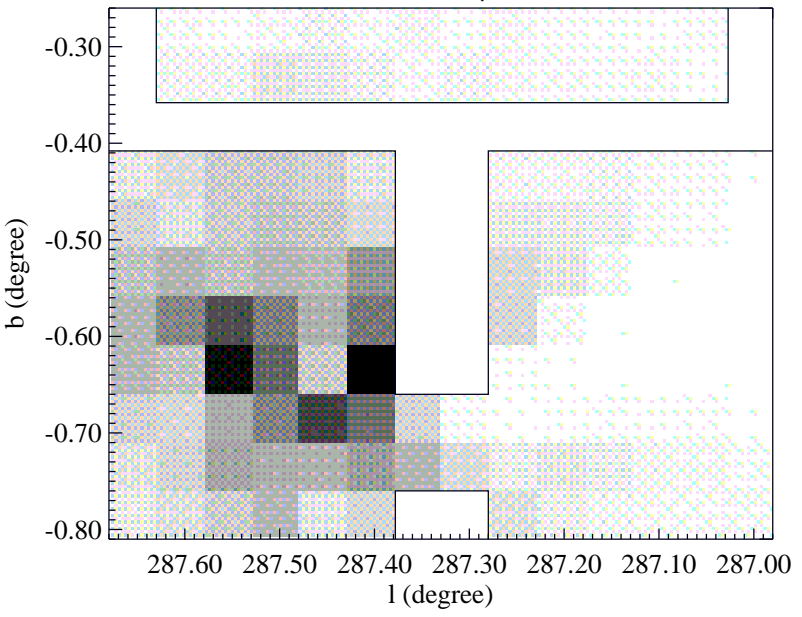

$\left[\mathrm{W} \mathrm{m} \mathrm{m}^{-2} \operatorname{str}^{-1}\right]$

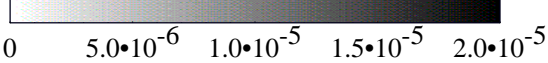

(d) $[\mathrm{NII}] 122 \mu \mathrm{m}$

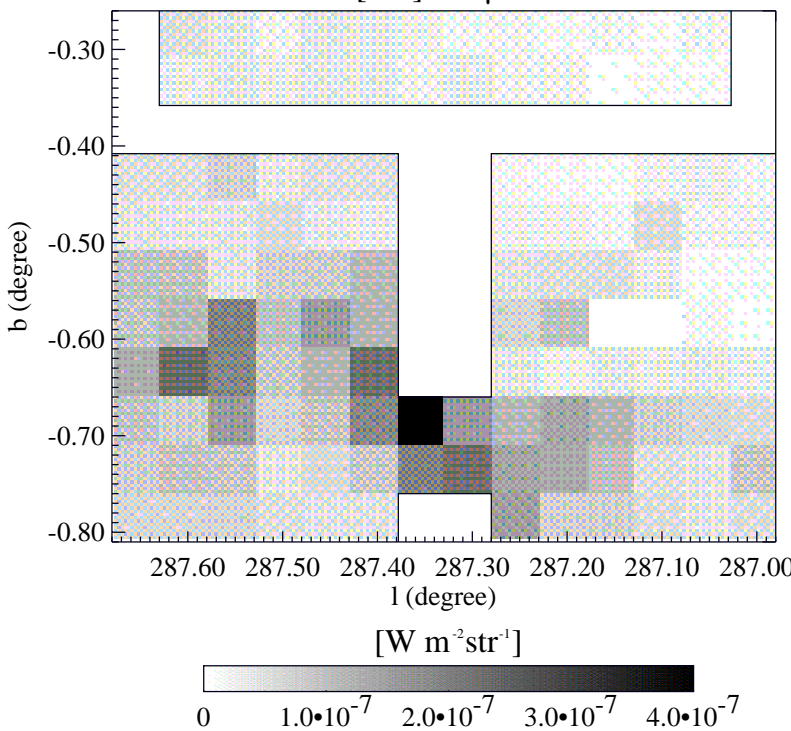

Fig. 3. The intensity distribution maps of the observed lines plotted in Galactic coordinates: a) [O III $52 \mu \mathrm{m}, \mathbf{b})[\mathrm{O}$ III] $88 \mu \mathrm{m}$, c) $[\mathrm{N} \mathrm{III}] 57 \mu \mathrm{m}$, and d) [N II $] 122 \mu \mathrm{m}$.

similar spectral characteristics, but its brightness is about $1 / 10$ of the optically bright region (Fig. 2 b). In the direction to the molecular cloud region, in which strong absorption has been observed in optical wavelengths, the continuum peaks at longer wavelengths and the [O III] and [N III] line intensities become weaker than in the optically bright region (Fig. 2c).

Figure 3 shows the intensity maps of the [O III] $52 \mu \mathrm{m}$, $88 \mu \mathrm{m},[\mathrm{N}$ III] $57 \mu \mathrm{m}$, and [N II] $122 \mu \mathrm{m}$ lines. The white T-shaped area enclosed by solid lines in the figures indicates the positions where the observations were not executed. For four positions at $(l, b)=\left(287^{\circ} 4,-0.63\right)$, $\left(287^{\circ} .4,-0.68\right),\left(287^{\circ} .25,-0.63\right)$, and $\left(287^{\circ} .25,-0.68\right)$, the observations were executed twice and the averaged values were adopted for the line intensities. The [N III] $57 \mu \mathrm{m}$ line shows a very similar distribution to that of the [O III] lines. This can easily be understood since $\mathrm{O}^{2+}$ and $\mathrm{N}^{2+}$ have similar ionization potentials (35.1 eV and $29.6 \mathrm{eV})$. Their emission has two peaks; one at the center of Car I $\left(l=287^{\circ} .405, b=-0.637\right)$ and the other at the center of Car II $\left(l=287^{\circ} .555, b=-0.636\right)$. The bright IR star, $\eta$ Car, is located in the grid next east of the Car II peak and it was observed within the LWS01 $80^{\prime \prime}$ beam at the grid position. Car I is believed to be excited by Tr 14 OB stars and there is an ionization front near the border between the $\mathrm{H}$ II region and the molecular cloud. The Car I peak of $[\mathrm{O}$ III $]$ and $[\mathrm{N} \mathrm{III}]$ lines is located near the border.

Figure 4 plots the [N III] $57 \mu \mathrm{m}$ intensity against the [O III] $52 \mu \mathrm{m}$ intensity. It is clearly seen that both intensities are proportional to each other fairly well for all 
Table 1. Intensity of the observed fine-structure emission and the electron density derived from the ratio of two [O III] lines.

\begin{tabular}{|c|c|c|c|c|c|c|c|c|c|c|c|c|}
\hline \multicolumn{2}{|c|}{ position } & \multicolumn{6}{|c|}{ Line intensities $\left(10^{-8} \mathrm{~W} \mathrm{~m}^{-2} \mathrm{sr}^{-1}\right)^{a}$} & & & \multirow{2}{*}{\multicolumn{2}{|c|}{$\begin{array}{l}\text { Electron density } \\
\left(\mathrm{cm}^{-3}\right)\end{array}$}} & \multirow{3}{*}{ Comments $^{c}$} \\
\hline$l$ & $b$ & [O III $]$ & $52 \mu \mathrm{m}$ & {$[\mathrm{O}$ iІ] } & $88 \mu \mathrm{m}$ & {$[\mathrm{N}$ III $]$} & $57 \mu \mathrm{m}$ & {$[\mathrm{N}$ II $]$} & $122 \mu \mathrm{m}$ & & & \\
\hline 287.356 & -0.736 & 239.3 & $(7.6)$ & 324.3 & $(10.0)$ & 100.8 & $(9.1)$ & 11.2 & $(1.5)$ & 59 & $(70,48)$ & \\
\hline 287.406 & -0.736 & 314.1 & $(77.0)$ & 427.5 & $(10.8)$ & 113.8 & $(2.2)$ & 4.4 & $(0.8)$ & 58 & $(122,0)$ & \\
\hline 287.355 & -0.686 & 154.1 & $(9.2)$ & 193.2 & $(6.7)$ & 62.0 & $(3.4)$ & 22.5 & $(4.5)$ & 80 & $(100,61)$ & \\
\hline 287.405 & -0.686 & 731.1 & $(19.2)$ & 515.7 & (129.8) & 204.6 & $(10.0)$ & 10.2 & (1.5) & 315 & $(465,175)$ & \\
\hline 287.406 & -0.787 & 123.7 & (13.6) & 186.6 & $(2.7)$ & 44.2 & $(1.6)$ & 2.7 & $(0.5)$ & 33 & $(59,8)$ & \\
\hline 287.456 & -0.786 & 149.8 & $(3.8)$ & 116.1 & $(17.8)$ & 39.1 & $(1.7)$ & 2.6 & $(0.6)$ & 264 & $(345,187)$ & \\
\hline 287.506 & -0.786 & 251.9 & $(22.4)$ & 315.6 & $(21.1)$ & 87.7 & $(1.7)$ & 2.9 & $(0.7)$ & 80 & $(112,49)$ & \\
\hline 287.556 & -0.786 & 223.5 & $(5.1)$ & 222.2 & (17.9) & 79.6 & $(4.7)$ & 3.9 & $(0.9)$ & 156 & $(187,125)$ & \\
\hline 287.606 & -0.786 & 66.8 & $(14.0)$ & 136.7 & $(4.0)$ & 29.6 & $(3.3)$ & 3.7 & $(0.7)$ & $<9$ & & \\
\hline 287.656 & -0.786 & 75.3 & $(2.4)$ & 92.8 & $(0.7)$ & 22.3 & $(1.0)$ & 3.7 & $(0.8)$ & 85 & $(94,76)$ & \\
\hline 287.456 & -0.736 & 235.9 & $(30.5)$ & 345.4 & $(5.3)$ & 84.3 & $(2.6)$ & 3.4 & (1.3) & 40 & $(71,9)$ & \\
\hline 287.506 & -0.736 & 412.9 & $(8.7)$ & 357.2 & $(28.4)$ & 86.7 & $(9.2)$ & 2.5 & (1.0) & 212 & $(249,176)$ & \\
\hline 287.556 & -0.736 & 335.7 & (19.5) & 399.7 & $(7.0)$ & 97.7 & $(5.6)$ & 5.0 & $(0.9)$ & 95 & $(114,77)$ & \\
\hline 287.606 & -0.736 & 156.8 & $(3.0)$ & 204.3 & $(1.7)$ & 56.4 & $(2.5)$ & 5.3 & $(0.8)$ & 69 & $(75,64)$ & \\
\hline 287.656 & -0.736 & 102.9 & (3.6) & 132.6 & $(2.9)$ & 33.5 & $(1.3)$ & 4.3 & (1.6) & 72 & $(84,61)$ & \\
\hline 287.455 & -0.686 & 1018.0 & $(39.9)$ & 831.2 & $(33.4)$ & 263.0 & $(5.4)$ & 5.2 & (1.7) & 238 & $(265,212)$ & \\
\hline 287.505 & -0.686 & 624.4 & $(26.9)$ & 545.2 & $(21.1)$ & 145.8 & $(6.3)$ & 3.9 & (1.0) & 208 & $(233,183)$ & \\
\hline 287.555 & -0.686 & 516.0 & $(6.6)$ & 371.3 & $(27.6)$ & 110.0 & $(16.3)$ & 8.9 & (1.1) & 303 & $(346,262)$ & \\
\hline 287.605 & -0.686 & 216.4 & (8.9) & 180.5 & (13.3) & 70.2 & $(1.7)$ & 4.3 & $(0.6)$ & 228 & $(268,190)$ & \\
\hline 287.655 & -0.686 & 155.8 & (4.8) & 202.6 & $(5.0)$ & 53.6 & $(1.2)$ & 5.3 & (0.6) & 70 & $(81,59)$ & \\
\hline 287.405 & -0.637 & 1572.0 & $(41.7)$ & 1114.0 & $(7.1)$ & 401.2 & $(13.0)$ & 12.7 & (2.7) & 312 & $(328,297)$ & \\
\hline 287.455 & -0.636 & 469.8 & (15.0) & 238.2 & $(38.6)$ & 134.2 & $(5.7)$ & 6.5 & (1.5) & 553 & $(707,410)$ & \\
\hline 287.505 & -0.636 & 769.1 & $(24.1)$ & 675.0 & $(4.3)$ & 190.0 & $(13.2)$ & 4.8 & (1.3) & 206 & $(220,192)$ & \\
\hline 287.555 & -0.636 & 1572.0 & $(34.4)$ & 1071.0 & $(7.0)$ & 399.4 & $(8.3)$ & 10.8 & (1.9) & 335 & $(349,321)$ & \\
\hline 287.605 & -0.636 & 468.7 & (206.3) & 265.1 & $(8.7)$ & $<62.9$ & & 13.2 & (3.5) & 462 & $(835,149)$ & $\eta \mathrm{Car}$ \\
\hline 287.655 & -0.636 & 252.5 & $(13.6)$ & 312.4 & (11.3) & 82.5 & $(7.5)$ & 7.4 & $(1.2)$ & 84 & $(103,65)$ & \\
\hline 287.405 & -0.586 & 477.5 & (53.7) & 596.0 & (10.8) & 173.5 & $(5.9)$ & 6.4 & (2.1) & 81 & $(114,49)$ & \\
\hline 287.455 & -0.586 & 367.7 & $(36.7)$ & 353.2 & $(26.0)$ & 111.0 & $(3.4)$ & 8.9 & (1.6) & 169 & $(217,121)$ & \\
\hline 287.505 & -0.586 & 677.8 & $(25.3)$ & 571.9 & (14.1) & 158.8 & $(4.4)$ & 5.6 & $(1.2)$ & 223 & $(244,203)$ & \\
\hline 287.555 & -0.586 & 915.2 & $(23.3)$ & 773.6 & $(8.5)$ & 255.8 & $(8.9)$ & 11.1 & (1.9) & 222 & $(235,210)$ & \\
\hline 287.605 & -0.586 & 532.2 & $(5.1)$ & 535.0 & $(10.3)$ & 80.0 & $(15.8)$ & 6.2 & (1.4) & 151 & $(159,144)$ & \\
\hline 287.655 & -0.586 & 363.2 & (4.4) & 330.2 & $(23.1)$ & 101.2 & $(8.6)$ & 5.0 & (1.0) & 191 & $(220,161)$ & \\
\hline 287.405 & -0.536 & 409.7 & $(56.3)$ & 467.9 & (13.5) & 148.8 & $(9.1)$ & 6.2 & $(2.2)$ & 108 & $(153,64)$ & \\
\hline 287.455 & -0.536 & 325.1 & $(9.9)$ & 297.4 & (13.2) & 85.8 & $(4.8)$ & 4.5 & (1.6) & 188 & $(210,166)$ & \\
\hline 287.505 & -0.536 & 289.8 & (7.4) & 318.8 & $(1.9)$ & 81.8 & (1.4) & 4.3 & $(0.9)$ & 120 & $(129,111)$ & \\
\hline 287.555 & -0.536 & 183.7 & $(9.2)$ & 265.7 & $(7.6)$ & 59.4 & $(4.0)$ & 2.6 & $(0.9)$ & 43 & $(57,29)$ & \\
\hline 287.605 & -0.536 & 309.0 & (3.4) & 330.4 & $(14.1)$ & 94.0 & $(3.0)$ & 5.3 & (0.9) & 130 & $(145,115)$ & \\
\hline 287.655 & -0.536 & 307.8 & (7.9) & 262.9 & $(23.6)$ & 75.1 & (11.6) & 5.4 & (1.3) & 218 & $(260,176)$ & \\
\hline 287.405 & -0.486 & 132.9 & (7.4) & 168.9 & $(5.5)$ & 32.6 & $(1.6)$ & 2.0 & $(0.7)$ & 76 & $(94,58)$ & \\
\hline 287.455 & -0.486 & 236.1 & (6.4) & 240.2 & $(5.9)$ & 58.1 & $(3.2)$ & 2.3 & $(0.7)$ & 147 & $(160,134)$ & \\
\hline 287.505 & -0.486 & 240.5 & (7.6) & 258.9 & (10.7) & 67.1 & $(4.4)$ & 3.3 & $(0.9)$ & 127 & $(145,110)$ & \\
\hline 287.555 & -0.486 & 203.7 & $(10.2)$ & 223.8 & $(5.9)$ & 47.1 & $(3.4)$ & 2.5 & (0.4) & 121 & $(139,102)$ & \\
\hline 287.605 & -0.486 & 105.1 & $(1.3)$ & 127.5 & $(4.6)$ & 32.8 & $(1.7)$ & 2.4 & $(0.5)$ & 90 & $(101,78)$ & \\
\hline 287.655 & -0.486 & 139.1 & (3.6) & 191.6 & $(7.3)$ & 44.5 & $(1.0)$ & 2.6 & $(0.5)$ & 55 & $(67,43)$ & \\
\hline 287.405 & -0.436 & 61.3 & $(7.3)$ & 115.3 & $(3.4)$ & 30.3 & $(1.8)$ & 2.8 & $(0.7)$ & $<10$ & & \\
\hline 287.455 & -0.436 & 186.1 & (5.8) & 205.6 & (1.3) & 60.4 & $(3.6)$ & 2.9 & $(0.8)$ & 119 & $(129,108)$ & \\
\hline 287.505 & -0.436 & 201.8 & (4.7) & 231.3 & $(7.0)$ & 52.8 & $(3.6)$ & 2.2 & $(0.7)$ & 107 & $(119,95)$ & \\
\hline 287.555 & -0.436 & 145.0 & $(16.8)$ & 228.1 & (1.3) & 62.3 & $(2.7)$ & 4.2 & (1.0) & 24 & $(49,0)$ & \\
\hline 287.605 & -0.436 & 128.0 & $(2.8)$ & 158.0 & $(4.1)$ & 36.9 & $(1.4)$ & 3.0 & $(0.7)$ & 85 & $(94,75)$ & \\
\hline 287.655 & -0.436 & 89.0 & (3.0) & 108.8 & $(6.8)$ & 26.9 & $(1.7)$ & 3.0 & $(0.4)$ & 87 & $(108,67)$ & \\
\hline 287.256 & -0.737 & 74.5 & (3.8) & 89.3 & $(10.4)$ & 37.6 & $(3.2)$ & 10.1 & (1.9) & 93 & $(132,56)$ & \\
\hline 287.306 & -0.736 & 119.8 & (4.7) & 160.3 & $(4.6)$ & 52.7 & $(3.2)$ & 12.8 & (1.6) & 62 & $(75,50)$ & \\
\hline 287.255 & -0.687 & 4.5 & (1.5) & 16.2 & $(0.7)$ & $<4.9$ & & 6.8 & $(1.4)$ & - & & $\mathrm{CO}$ \\
\hline 287.305 & -0.686 & 15.4 & (3.7) & 30.1 & $(0.9)$ & $<7.5$ & & 9.1 & (3.0) & $<23$ & & $\mathrm{CO}$ \\
\hline 287.006 & -0.788 & 32.3 & (1.7) & 42.9 & $(0.5)$ & 18.3 & $(1.2)$ & 3.0 & $(0.3)$ & 65 & $(79,51)$ & \\
\hline 287.056 & -0.787 & 23.2 & (1.9) & 35.6 & (1.1) & 10.4 & $(0.6)$ & 2.4 & $(0.4)$ & 29 & $(49,10)$ & \\
\hline 287.107 & -0.786 & 29.8 & $(4.1)$ & 40.2 & $(1.2)$ & 11.5 & $(1.6)$ & 3.1 & $(0.8)$ & 61 & $(98,24)$ & \\
\hline
\end{tabular}


Table 1. continued.

\begin{tabular}{|c|c|c|c|c|c|c|c|c|c|c|c|c|}
\hline \multicolumn{2}{|c|}{ position } & \multicolumn{8}{|c|}{ Line intensities $\left(10^{-8} \mathrm{~W} \mathrm{~m}^{-2} \mathrm{sr}^{-1}\right)$} & \multirow{2}{*}{\multicolumn{2}{|c|}{$\begin{array}{l}\text { Electron density } \\
\qquad\left(\mathrm{cm}^{-3}\right)\end{array}$}} & \multirow{3}{*}{ Comments } \\
\hline$l$ & $b$ & {$[\mathrm{O}$ III $]$} & $52 \mu \mathrm{m}$ & \multicolumn{2}{|c|}{$[\mathrm{O}$ III $] 88 \mu \mathrm{m}$} & \multicolumn{2}{|c|}{$[\mathrm{N}$ III $] 57 \mu \mathrm{m}$} & \multicolumn{2}{|c|}{$[\mathrm{N}$ II $] 122 \mu \mathrm{m}$} & & & \\
\hline 287.156 & -0.786 & 45.8 & $(3.8)$ & 51.6 & $(3.1)$ & 20.1 & $(2.6)$ & 4.3 & $(1.0)$ & 112 & $(145,80)$ & \\
\hline 287.207 & -0.785 & 60.8 & $(3.3)$ & 75.6 & $(2.5)$ & 24.3 & $(1.1)$ & 4.2 & $(1.0)$ & 82 & $(100,64)$ & \\
\hline 287.257 & -0.784 & 109.2 & $(3.2)$ & 136.1 & $(7.8)$ & 48.9 & $(1.5)$ & 8.6 & $(0.9)$ & 82 & $(100,64)$ & \\
\hline 287.006 & -0.738 & 16.0 & $(2.4)$ & 34.6 & $(1.4)$ & 15.5 & $(0.8)$ & 5.0 & $(0.5)$ & - & & \\
\hline 287.056 & -0.737 & 33.9 & $(2.0)$ & 47.0 & $(1.3)$ & 15.7 & $(1.2)$ & 2.5 & $(0.4)$ & 53 & $(69,37)$ & \\
\hline 287.106 & -0.736 & 40.3 & $(1.2)$ & 52.2 & $(0.5)$ & 18.1 & $(1.2)$ & 2.8 & $(0.7)$ & 71 & $(80,62)$ & \\
\hline 287.156 & -0.736 & 66.4 & (1.7) & 82.4 & $(1.1)$ & 38.1 & $(1.8)$ & 5.7 & $(1.2)$ & 83 & $(91,75)$ & \\
\hline 287.206 & -0.735 & 86.3 & $(2.5)$ & 112.2 & $(1.9)$ & 48.8 & $(2.0)$ & 7.6 & (1.3) & 70 & $(79,61)$ & \\
\hline 287.005 & -0.688 & 2.1 & $(0.8)$ & 10.6 & $(0.7)$ & 6.6 & $(1.4)$ & 3.2 & $(0.6)$ & - & & \\
\hline 287.055 & -0.687 & 14.5 & (1.5) & 23.6 & $(0.5)$ & 11.6 & $(1.1)$ & 3.6 & $(0.4)$ & 16 & $(39,0)$ & \\
\hline 287.105 & -0.686 & 18.0 & $(0.8)$ & 20.2 & $(1.4)$ & 11.0 & $(1.2)$ & 4.4 & $(0.8)$ & 113 & $(140,87)$ & \\
\hline 287.155 & -0.686 & 16.8 & $(3.3)$ & 23.1 & $(0.7)$ & 12.2 & $(2.4)$ & 6.6 & (1.0) & 56 & $(107,7)$ & \\
\hline 287.205 & -0.685 & $<12.1$ & & 18.5 & $(0.9)$ & 5.4 & $(1.6)$ & 8.0 & $(1.7)$ & $<30$ & & \\
\hline 287.004 & -0.638 & 3.6 & $(1.5)$ & 4.8 & $(0.5)$ & $<1.0$ & & 1.7 & $(0.5)$ & 62 & $(176,0)$ & \\
\hline 287.054 & -0.637 & 6.4 & (1.5) & 5.2 & $(0.5)$ & 3.8 & $(1.6)$ & 2.1 & $(0.5)$ & 238 & $(359,124)$ & clump \\
\hline 287.104 & -0.636 & $<5.9$ & & 4.2 & $(0.6)$ & 2.6 & $(0.9)$ & 1.8 & $(0.6)$ & $<310$ & & $\mathrm{CO}$ \\
\hline 287.154 & -0.636 & $<3.7$ & & 6.8 & $(0.7)$ & $<1.1$ & & 2.2 & $(0.8)$ & - & & $\mathrm{CO}$ \\
\hline 287.204 & -0.635 & $<1.7$ & & 9.0 & $(0.6)$ & $<1.1$ & & $<4.5$ & & - & & $\mathrm{CO}$ \\
\hline 287.254 & -0.634 & 17.2 & $(3.4)$ & 20.0 & $(1.5)$ & 5.2 & $(2.2)$ & $<4.9$ & & 103 & $(169,39)$ & \\
\hline 287.006 & -0.587 & 1.0 & $(0.3)$ & 3.3 & $(0.6)$ & $<3.4$ & & $<1.1$ & & - & & \\
\hline 287.056 & -0.587 & $<5.7$ & & $<5.3$ & & $<1.0$ & & $<2.2$ & & - & & \\
\hline 287.106 & -0.586 & $<2.2$ & & 4.2 & $(0.6)$ & $<0.8$ & & $<0.5$ & & - & & $\mathrm{CO}$ \\
\hline 287.156 & -0.586 & 4.5 & (1.3) & 8.6 & $(0.9)$ & $<0.8$ & & $<0.5$ & & $<40$ & & $\mathrm{CO}$ \\
\hline 287.206 & -0.585 & 32.5 & $(3.7)$ & 39.1 & $(3.4)$ & 7.9 & $(1.2)$ & 5.5 & $(2.1)$ & 92 & $(135,51)$ & \\
\hline 287.256 & -0.585 & 118.2 & $(15.1)$ & 184.8 & $(3.7)$ & 53.2 & $(1.9)$ & 3.9 & $(1.5)$ & 25 & $(54,0)$ & \\
\hline 287.006 & -0.537 & $<1.4$ & & 3.7 & $(0.8)$ & $<2.1$ & & 0.7 & $(0.2)$ & - & & \\
\hline 287.056 & -0.537 & 3.3 & (1.4) & 3.8 & $(0.3)$ & $<2.7$ & & $<1.6$ & & 99 & $(237,0)$ & \\
\hline 287.106 & -0.536 & 6.2 & $(2.7)$ & 6.6 & $(0.6)$ & $<1.3$ & & $<4.4$ & & 129 & $(286,0)$ & \\
\hline 287.156 & -0.536 & 26.8 & $(2.5)$ & 43.6 & $(1.1)$ & 12.2 & $(1.1)$ & 3.1 & $(0.7)$ & 16 & $(37,0)$ & \\
\hline 287.206 & -0.535 & 96.2 & (3.7) & 119.1 & $(5.1)$ & 32.3 & $(2.7)$ & 2.9 & $(0.8)$ & 84 & $(100,67)$ & \\
\hline 287.256 & -0.535 & 196.5 & (8.3) & 173.7 & (16.6) & 59.1 & $(2.8)$ & 2.7 & $(0.9)$ & 203 & $(248,158)$ & clump \\
\hline 287.005 & -0.487 & $<5.8$ & & 6.4 & $(1.0)$ & $<1.1$ & & 1.4 & $(0.3)$ & $<119$ & & \\
\hline 287.055 & -0.487 & 11.8 & (1.0) & 25.3 & $(1.3)$ & 6.6 & $(0.9)$ & 1.4 & $(0.3)$ & - & & \\
\hline 287.105 & -0.486 & 29.6 & $(2.9)$ & 33.2 & $(3.3)$ & 7.6 & $(0.8)$ & 3.2 & $(0.5)$ & 114 & $(160,69)$ & \\
\hline 287.155 & -0.486 & 58.6 & $(2.6)$ & 74.1 & $(5.8)$ & 17.9 & $(1.4)$ & 1.2 & $(0.5)$ & 78 & $(103,52)$ & \\
\hline 287.205 & -0.485 & 77.4 & $(3.5)$ & 91.9 & $(6.4)$ & 23.0 & $(1.8)$ & 1.5 & $(0.6)$ & 96 & $(121,71)$ & \\
\hline 287.255 & -0.485 & 83.4 & (1.4) & 99.0 & $(4.9)$ & 25.6 & $(0.9)$ & 1.5 & $(0.7)$ & 96 & $(112,80)$ & \\
\hline 287.005 & -0.437 & 8.4 & $(1.2)$ & 10.9 & $(0.5)$ & 3.7 & $(1.1)$ & 1.2 & $(0.2)$ & 70 & $(111,30)$ & \\
\hline 287.055 & -0.437 & 21.4 & (1.7) & 29.1 & $(2.3)$ & 8.2 & $(1.0)$ & 1.7 & $(0.4)$ & 58 & $(87,30)$ & \\
\hline 287.105 & -0.436 & 21.8 & $(2.2)$ & 31.7 & $(1.5)$ & 9.2 & $(2.4)$ & $<4.6$ & & 41 & $(68,15)$ & \\
\hline 287.155 & -0.436 & 45.4 & $(3.0)$ & 60.6 & $(0.6)$ & 14.1 & $(0.9)$ & 0.8 & $(0.3)$ & 63 & $(81,46)$ & \\
\hline 287.205 & -0.435 & 31.1 & (1.5) & 44.7 & $(3.3)$ & 9.6 & $(1.0)$ & 0.9 & $(0.4)$ & 44 & $(66,23)$ & \\
\hline 287.255 & -0.435 & 34.8 & (1.4) & 48.7 & $(2.8)$ & 12.7 & $(1.4)$ & 1.0 & $(0.3)$ & 51 & $(68,33)$ & \\
\hline 287.055 & -0.336 & 10.8 & $(0.8)$ & 19.4 & $(1.3)$ & 4.6 & $(1.5)$ & 0.7 & $(0.3)$ & $<16$ & & \\
\hline 287.105 & -0.336 & 22.0 & (2.7) & 31.2 & $(1.1)$ & 7.3 & $(1.0)$ & 0.8 & $(0.3)$ & 48 & $(80,17)$ & \\
\hline 287.155 & -0.336 & 26.5 & (1.7) & 29.3 & $(3.3)$ & 9.4 & $(1.0)$ & $<1.0$ & & 119 & $(162,76)$ & \\
\hline 287.206 & -0.336 & 17.9 & (1.7) & 32.8 & $(1.1)$ & 9.0 & $(0.8)$ & 1.4 & $(0.5)$ & $<12$ & & \\
\hline 287.256 & -0.336 & 27.8 & (1.8) & 41.2 & $(1.8)$ & 9.3 & $(1.0)$ & 1.4 & $(0.5)$ & 37 & $(55,19)$ & \\
\hline 287.306 & -0.336 & 40.2 & $(3.0)$ & 57.1 & $(1.6)$ & 17.8 & $(1.8)$ & 1.9 & $(0.4)$ & 47 & $(67,28)$ & \\
\hline 287.356 & -0.336 & 28.5 & (1.7) & 37.5 & $(1.4)$ & 6.0 & $(1.5)$ & 1.4 & $(0.3)$ & 67 & $(86,48)$ & \\
\hline 287.406 & -0.336 & 45.5 & $(2.2)$ & 65.3 & $(3.4)$ & 13.1 & $(1.9)$ & 2.0 & $(0.8)$ & 44 & $(62,27)$ & \\
\hline 287.456 & -0.336 & 74.6 & (1.4) & 99.6 & $(2.1)$ & 22.0 & $(2.5)$ & 2.2 & $(0.6)$ & 63 & $(70,55)$ & \\
\hline 287.506 & -0.336 & 72.5 & (5.1) & 117.5 & $(3.5)$ & 26.1 & $(1.6)$ & 1.8 & $(0.4)$ & 17 & $(33,1)$ & \\
\hline 287.556 & -0.336 & 49.5 & (6.2) & 60.1 & $(3.6)$ & 23.2 & $(2.0)$ & 2.2 & $(0.3)$ & 89 & $(130,49)$ & \\
\hline 287.606 & -0.335 & 29.5 & $(3.1)$ & 52.2 & $(2.5)$ & 13.4 & $(1.4)$ & 1.8 & $(0.2)$ & $<22$ & & \\
\hline 287.055 & -0.286 & 19.6 & $(2.4)$ & 28.1 & $(3.1)$ & 7.6 & $(2.1)$ & 1.2 & $(0.4)$ & 45 & $(85,6)$ & \\
\hline 287.105 & -0.286 & 12.9 & $(1.5)$ & 18.6 & $(2.3)$ & 6.1 & $(1.9)$ & 0.7 & $(0.2)$ & 43 & $(85,2)$ & \\
\hline 287.155 & -0.286 & 14.5 & $(2.1)$ & 23.7 & $(1.1)$ & 2.8 & $(0.5)$ & 1.2 & $(0.3)$ & 16 & $(48,0)$ & \\
\hline
\end{tabular}


Table 1. continued.

\begin{tabular}{|c|c|c|c|c|c|c|c|c|c|c|c|c|}
\hline \multicolumn{2}{|c|}{ position } & \multicolumn{8}{|c|}{ Line intensities $\left(10^{-8} \mathrm{~W} \mathrm{~m}^{-2} \mathrm{sr}^{-1}\right)$} & \multirow{2}{*}{\multicolumn{2}{|c|}{$\begin{array}{l}\text { Electron density } \\
\qquad\left(\mathrm{cm}^{-3}\right)\end{array}$}} & \multirow{3}{*}{ Comments } \\
\hline$l$ & $b$ & \multicolumn{2}{|c|}{ [O III] $52 \mu \mathrm{m}$} & \multicolumn{2}{|c|}{ [O III] $88 \mu \mathrm{m}$} & \multicolumn{2}{|c|}{$[\mathrm{N}$ III $] 57 \mu \mathrm{m}$} & \multicolumn{2}{|c|}{$[\mathrm{N}$ II $] 122 \mu \mathrm{m}$} & & & \\
\hline 287.205 & -0.286 & 20.5 & $(0.9)$ & 27.4 & $(1.7)$ & 6.1 & $(0.9)$ & 0.9 & $(0.4)$ & 62 & $(82,43)$ & \\
\hline 287.255 & -0.286 & 27.6 & $(1.4)$ & 43.3 & $(0.9)$ & 11.4 & $(1.1)$ & 1.3 & $(0.5)$ & 24 & $(37,12)$ & \\
\hline 287.305 & -0.286 & 20.2 & $(1.3)$ & 24.1 & $(2.3)$ & 7.4 & $(1.2)$ & 1.1 & $(0.4)$ & 94 & $(128,60)$ & \\
\hline 287.355 & -0.286 & 31.1 & $(2.1)$ & 50.1 & $(2.3)$ & 10.7 & $(1.2)$ & 1.5 & $(0.5)$ & 19 & $(36,2)$ & \\
\hline 287.405 & -0.286 & 40.0 & $(2.4)$ & 37.0 & $(4.8)$ & 13.1 & $(1.1)$ & 2.0 & $(0.8)$ & 184 & $(243,127)$ & clump \\
\hline 287.455 & -0.286 & 44.8 & $(2.6)$ & 64.3 & $(3.5)$ & 14.7 & $(1.2)$ & 2.2 & $(0.5)$ & 44 & $(64,25)$ & \\
\hline 287.505 & -0.286 & 22.7 & $(2.3)$ & 35.8 & $(1.4)$ & 7.0 & $(0.9)$ & 1.3 & $(0.3)$ & 23 & $(47,0)$ & \\
\hline 287.555 & -0.286 & 14.8 & $(1.6)$ & 24.8 & $(0.8)$ & 4.9 & $(0.9)$ & 2.3 & $(0.4)$ & 10 & $(34,0)$ & \\
\hline 287.605 & -0.285 & 25.9 & $(1.1)$ & 30.6 & $(2.3)$ & 10.1 & $(0.9)$ & 3.0 & $(0.2)$ & 97 & $(124,71)$ & \\
\hline
\end{tabular}

${ }^{a}$ The numbers in the parentheses indicate the errors $(1 \sigma)$, in which the absolute error of $30 \%$ is not included.

${ }^{b}$ The numbers in the parentheses indicate the upper and lower limits of the estimated electron density. The value of zero indicates that the lower limit is not constrained. Only the upper limit is given when the line ratio is less than the theoretical limit.

${ }^{c}$ The last column indicates the position containing $\eta$ Car, the position with strong CO emission (CO), and the positions with relatively large electron density in the molecular and surrounding regions (clump) (see text).

the observed positions except for the position near $\eta$ Car, where the $[\mathrm{N}$ III] line was not detected (indicated by an open circle). The emission at this point has strong continuum. The linear slope of Fig. 4 gives the intensity ratio of [N III] $57 \mu \mathrm{m}$ to [O III] $52 \mu \mathrm{m}$ of approximately 0.27 for the optically bright region. The other two regions have steeper slopes (see Sect. 4.4).

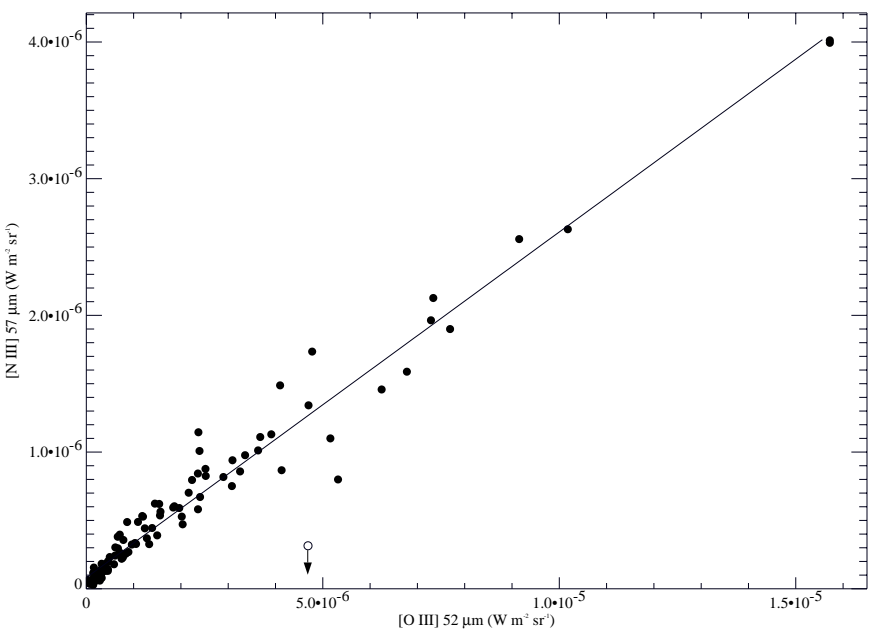

Fig. 4. The correlation of the [N III] $57 \mu \mathrm{m}$ and [O III] $52 \mu \mathrm{m}$ line intensities. The open circle represents the data of an upper limit of [N III $57 \mu \mathrm{m}$ line at the position of $\eta$ Car.

The spatial distribution of [O III] and [N III] ionic lines indicates that there is an ionized region enclosing Car I and Car II. However, these lines have also been detected clearly outside the optically bright H II regions. They are observed even in the dust lane in the western side of the observed area which shows strong absorption in visual wavelengths, indicating the presence of extended ionized gas around the Carina nebula.

The intensity map of the [N II] $122 \mu \mathrm{m}$ line (Fig. 3d) shows a slightly different spatial distribution. It peaks at the center of Car I and II, but the strongest emission is seen at the center of the observed region, $(l, b) \sim$ $(287.35,-0.67)$, around the border of the $\mathrm{H}$ II region and the molecular cloud. It is also detected clearly in the surrounding region, supporting the existence of the extended ionized gas around the Carina nebula.

Figure 5 shows the spectra of [O III] $88 \mu \mathrm{m}$ and [N III] $122 \mu \mathrm{m}$ observed at 4 positions away from the mapped area. The derived intensities and upper limits are listed in Table 2. For [O III] $88 \mu \mathrm{m}$ of these positions we use the data of the LW1 channel because of its higher signalto-noise ratio than that of the SW5. At two higher latitude positions $(b=0$.831), 3 of 4 scans clearly indicate the presence of [O III $] 88 \mu \mathrm{m}$ line and the detection seems secure. At lower latitude positions $\left(b=00^{\circ} 498\right)$ the forward scan data indicate the presence of [O III] line, but the backward scans do not. Therefore we conclude that the detection is uncertain and give the upper limits. The spectra at two positions indicate a marginal detection of [N II] $122 \mu \mathrm{m}$ line $(\sim 2.5 \sigma)$. The detection and the intensity at these positions should be taken with caution. The [O III] $52 \mu \mathrm{m}$ and [N III] $57 \mu \mathrm{m}$ lines were not detected at these positions and their upper limits are estimated to be $1 \times 10^{-8} \mathrm{~W} \mathrm{~m}^{-2} \mathrm{sr}^{-1}$. The detection of [O III] $88 \mu \mathrm{m}$ (and possibly [N II] $122 \mu \mathrm{m}$ ) at these positions supports the extended nature of the ionized gas.

Table 2. Line intensity in the higher Galactic latitude region.

\begin{tabular}{cccccc}
\hline \multicolumn{2}{c}{ position } & \multicolumn{3}{c}{ Line intensities } & $\left(10^{-9} \mathrm{~W} \mathrm{~m}^{-2} \mathrm{sr}^{-1}\right)$ \\
$l$ & $b$ & {$[$ O III] } & $88 \mu \mathrm{m}$ & {$[\mathrm{N} \mathrm{II}] 122 \mu \mathrm{m}$} \\
\hline 287.306 & 0.498 & $<19.4$ & & $<1.1$ & \\
287.356 & 0.498 & $<4.7$ & & 4.6 & $(1.9)$ \\
287.306 & 0.831 & 10.0 & $(3.0)$ & $<3.5$ & \\
287.356 & 0.831 & 11.0 & $(2.8)$ & 1.4 & $(0.5)$ \\
\hline
\end{tabular}


(a) [OIII] $88 \mu \mathrm{m}$

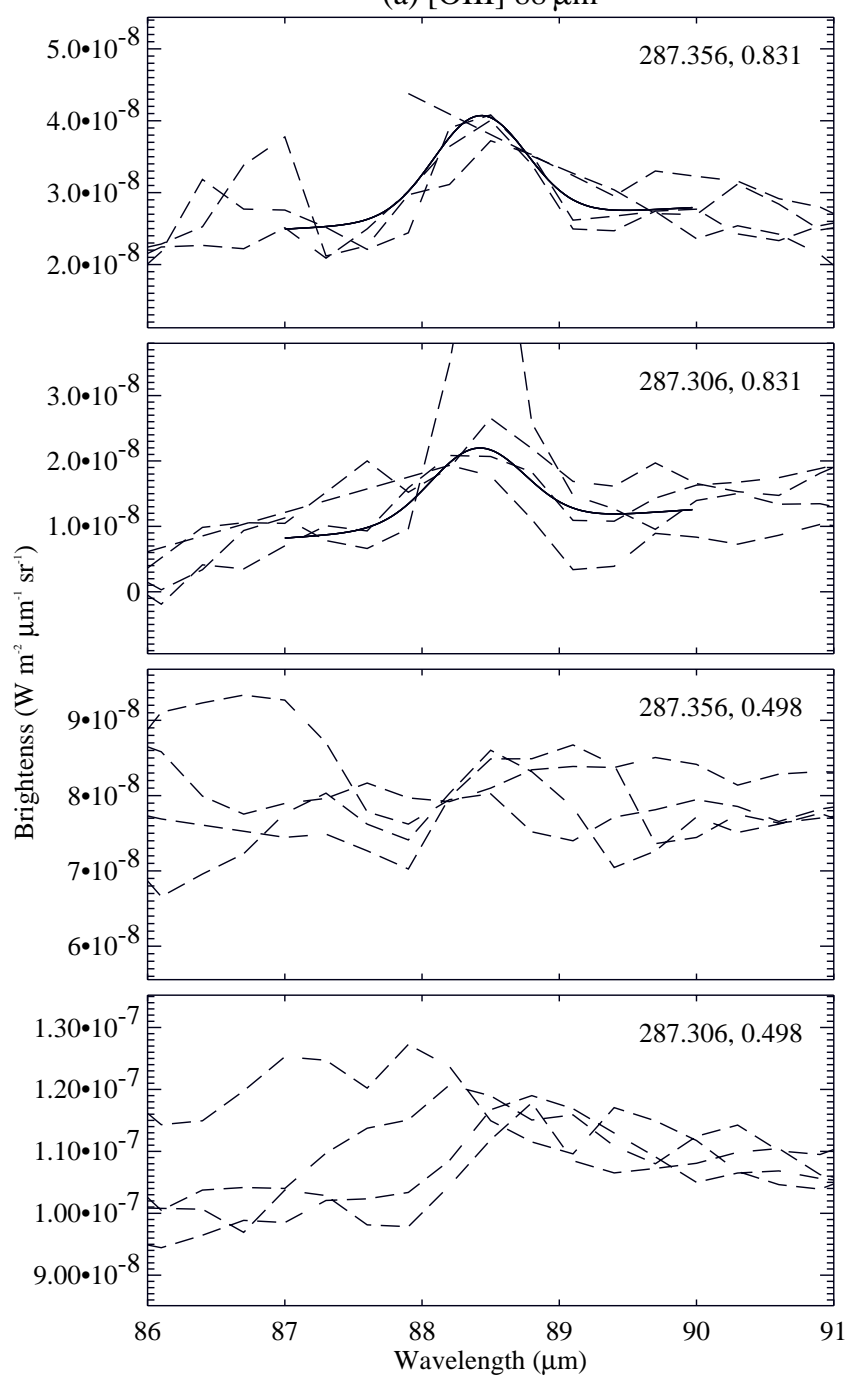

(b) $[\mathrm{NII}] 122 \mu \mathrm{m}$

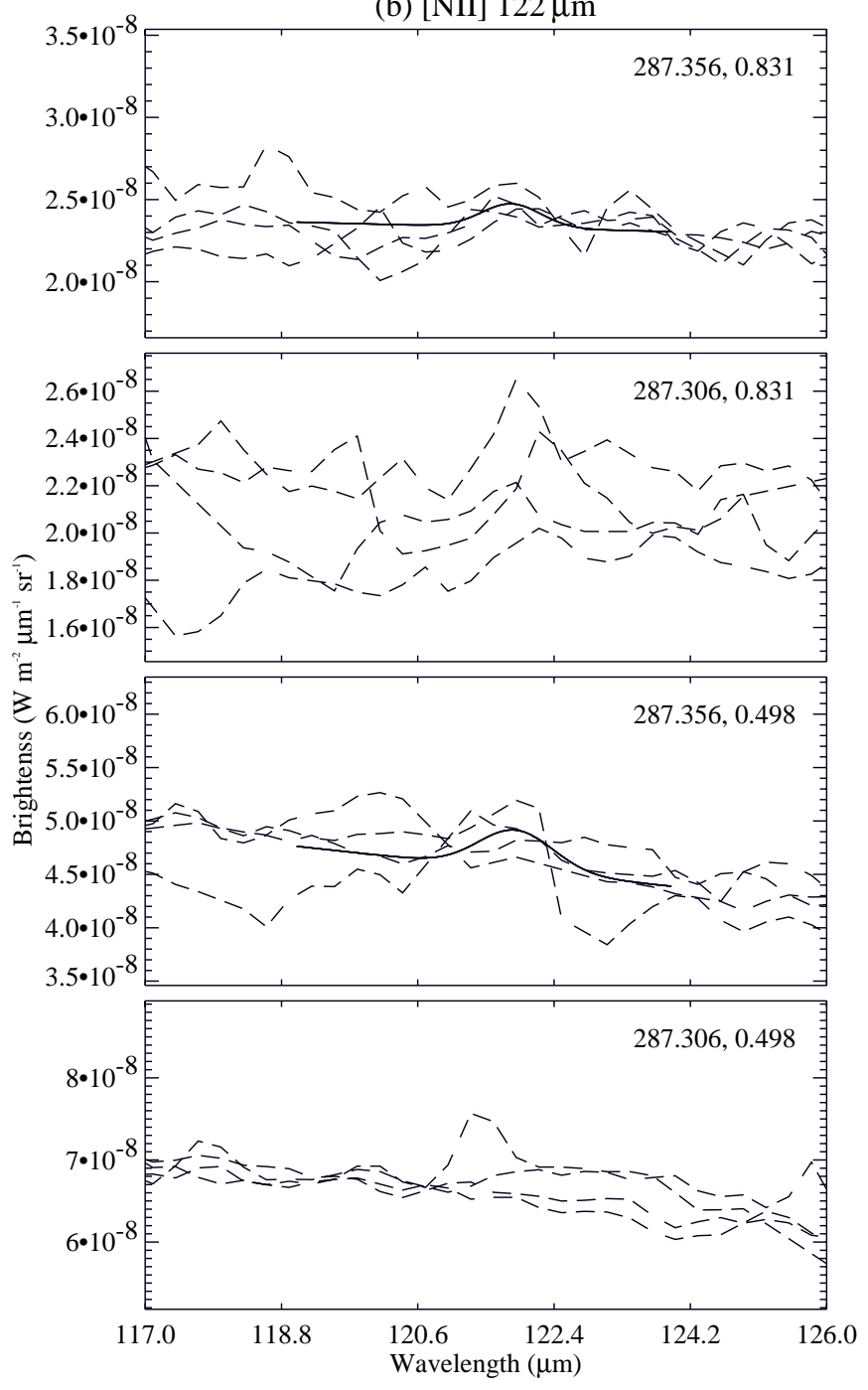

Fig. 5. Spectra at the high Galactic latitude positions: a) [O III] $88 \mu \mathrm{m}$, b) [N II] $122 \mu \mathrm{m}$. The spectral ranges are covered with 4 scans and each dashed line shows the independent scan. The solid lines indicate the fitted profiles for the lines whose detection is considered to be secure or marginal (see text).

\subsection{The electron density}

The electron density of the ionized gas can be derived from the ratio of the two [O III] lines (e.g., Moorwood et al. 1980). If the lines are optically thin, the line intensity ratio is given by

$R=\frac{A_{21} h \nu_{21} n_{2}\left(n_{\mathrm{e}}, T_{\mathrm{e}}\right)}{A_{10} h \nu_{10} n_{1}\left(n_{\mathrm{e}}, T_{\mathrm{e}}\right)}$

where $A_{21}$ and $A_{10}$ are the Einstein coefficients for spontaneous radiative transition, and $n_{2}$ and $n_{1}$ are the upper level populations for $52 \mu \mathrm{m}$ and $88 \mu \mathrm{m}$ emissions, respectively. The fractional populations can be calculated as a function of temperature and density of electron, ignoring the small effect of stimulated emission (Simpson 1975).

Figure 6 shows the line ratios calculated by Eq. (1) for $T_{\mathrm{e}}=5000 \mathrm{~K}, 7500 \mathrm{~K}$, and $20000 \mathrm{~K}$. The ratio does not sensitively depend on the assumed gas temperature for the low electron density $\left(n_{\mathrm{e}} \leq 100 \mathrm{~cm}^{-3}\right)$. For the collisional coefficients, we adopted the values calculated by
Aggarwal et al. (1982). We calculate the electron density assuming the gas temperature of $7500 \mathrm{~K}$ as a typical value for $\mathrm{H}$ il regions.

In Fig. 7 the histogram of the $[\mathrm{O} \mathrm{III}]$ line ratio is plotted for three regions separately. The errors in the line ratio are less than $15 \%$ for the optically bright ionized region (a) and the surrounding region (c) except for the $\eta$ Car position. The errors are typically $20-30 \%$ for the molecular cloud region (b). The optically bright ionized region has relatively large ratios compared to the other two regions, which are in the range $0.4-2.0$. About half of the positions in this region have the electron densities between 100 and $300 \mathrm{~cm}^{-3}$, corresponding to the ratio of $0.85-1.4$.

The derived electron density map is shown in Fig. 8 and the values are listed in Table 1 . In the electron density column, the upper and lower limits are also indicated in parentheses and take into account the uncertainty in the line ratio. For the surrounding region, the ratio ranges $0.5-1.1$. For about a half of the positions in this region 


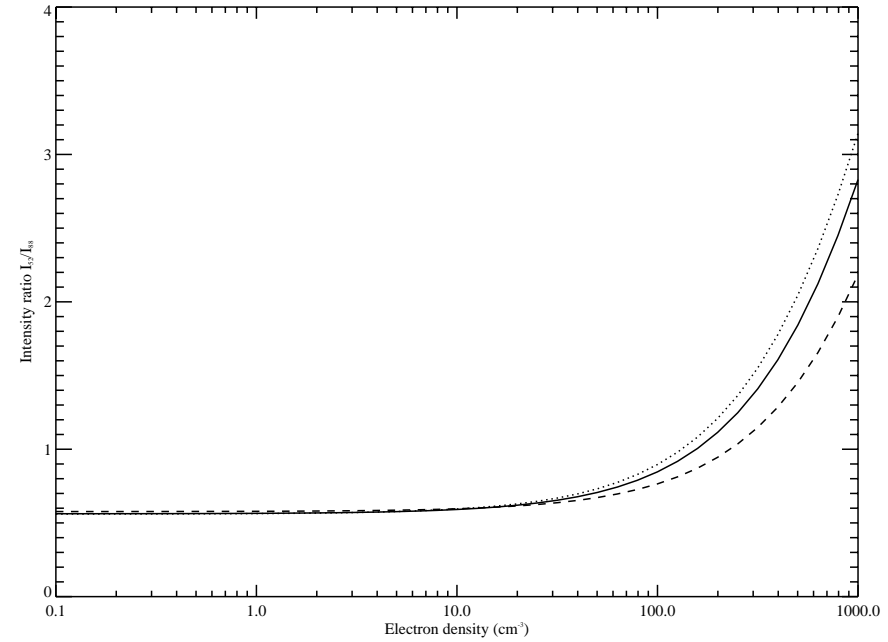

Fig. 6. The intensity ratio of the [O III $] 52 \mu \mathrm{m}$ to [O III $] 88 \mu \mathrm{m}$. The dotted line shows the ratio for $T_{\mathrm{e}}=5000 \mathrm{~K}$, the dashed line for $20000 \mathrm{~K}$ and the solid line for $7500 \mathrm{~K}$.

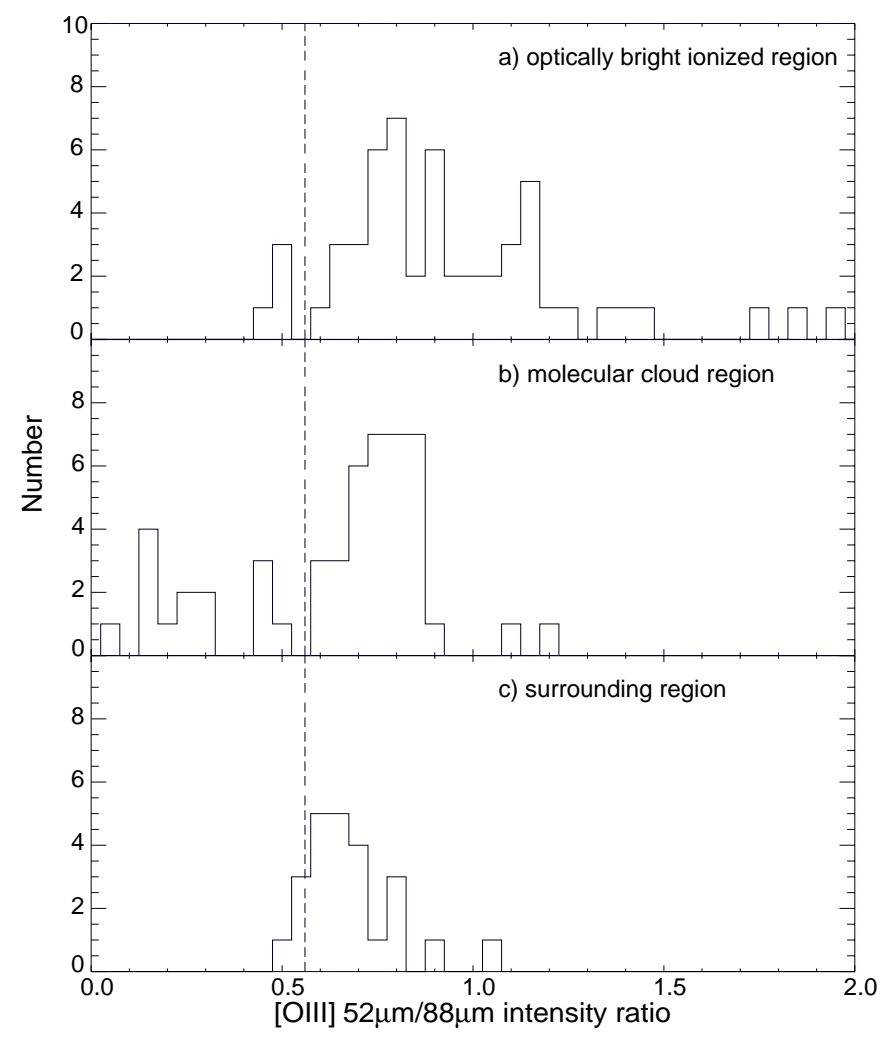

Fig. 7. The histogram of the distribution of [O III] intensity ratio. The dotted line indicates the theoretical lower limit of the ratio, assuming the electron temperature of $7500 \mathrm{~K}$. a) For the optically bright ionized region $\left(l>287^{\circ} .3, b<-0.4\right)$; b) for the molecular cloud region $\left(l<287^{\circ} .3, b<-0^{\circ} .4\right)$; c) for the surrounding region $(b>-0.4)$.

the ratio is just above the low density limit (0.57), and the electron density of these positions cannot be well determined. Except for two positions the electron density in this region is less than $100 \mathrm{~cm}^{-3}$ (see Table 1) and the mean electron density is derived to be $\sim 50 \mathrm{~cm}^{-3}$.

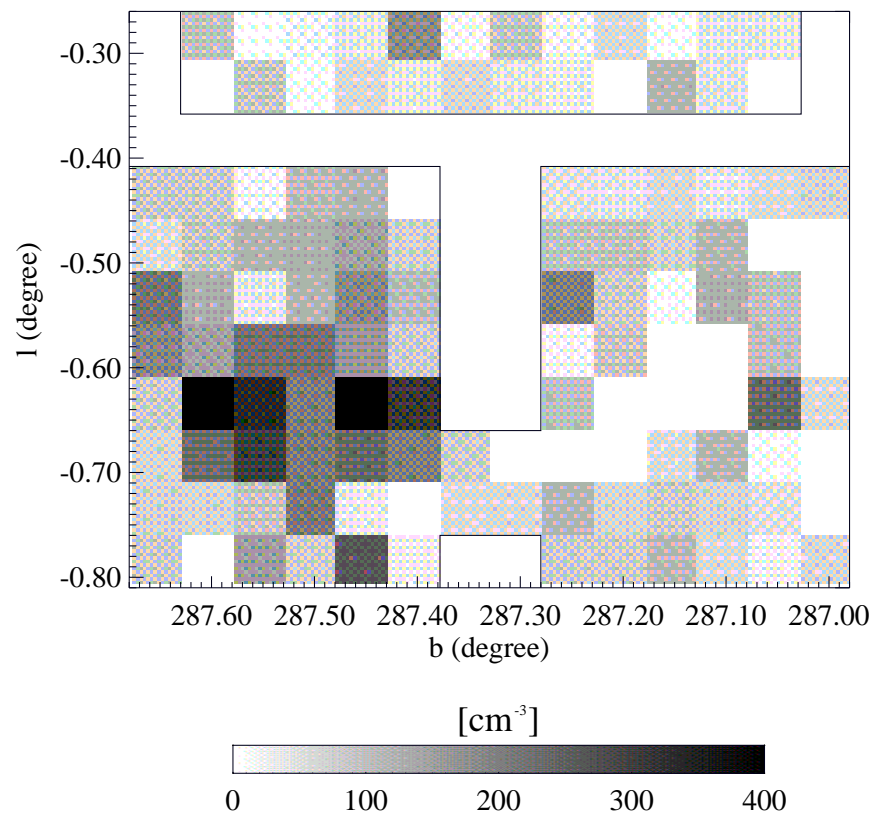

Fig. 8. The electron density distribution derived from the intensity ratio of the [O III] $52 \mu \mathrm{m}$ to the $88 \mu \mathrm{m}$ line. White color pixels indicate the regions where the line ratio is lower than the theoretical limit and the electron density cannot be derived (see text).

The low electron density gas is also seen both in the molecular cloud and optically bright regions. The [O III] $52 \mu \mathrm{m}$ to $88 \mu \mathrm{m}$ intensity ratio is lower than the theoretical lower limit 0.57 at 19 observed positions. The lower tail (the ratio of $0.40-0.57$ ) of the histogram in Fig. 7 can be attributed to the measurement errors. Some of the positions show a significantly low line ratio $(<0.4)$. All of them correspond to the positions with weak intensities and the low ratio may be ascribed to the measurement errors. We examine possible effects of the calibration errors and the interstellar extinction below.

The derived electron density depends primarily on the relative calibration between the corresponding bands, but not on the absolute calibration. The discrepancy between adjacent detectors seen in Fig. 2 can be attributed to the uncertainties either in the sensitivity, the spatial brightness distribution in the beam, or the dark current of each detector. In case of bright objects like the Carina nebula, the uncertainties in the sensitivity and/or the brightness distribution in the beam are supposed to be dominant compared to the dark current uncertainty. The uncertainties can be estimated from the gaps in the continuum flux. If the continuum level of the channel for [O III] $88 \mu \mathrm{m}$ (SW5) is scaled to that for [O III] $52 \mu \mathrm{m}$ (SW2) to correct the gaps, the scaling factor ranges 1.0-1.3, depends on the line of sight, except for the positions of $\eta$ Car and $(l, b)=\left(287^{\circ} .055,-0.286\right)$. At the latter position, the factor is 0.98 and the scaling will not affect the results. Therefore, the line ratio would further be reduced and the corresponding electron density would be decreased if we apply the scaling. In the present analysis we do not correct 
the gaps between the detectors and thus the present results should be upper limits in this sense.

The correction for extended sources increases the [O III] $52 \mu \mathrm{m}$ to $88 \mu \mathrm{m}$ ratio by a factor of 1.28 . Compared to the original data, this correction increases the electron density. It is unlikely that the errors in the extended source correction factor affect the [O III] line ratio largely because the wavelengths of the two lines are not significantly different.

The uncertainty in the collisional coefficients affects the estimation of the electron density. In the low density limit, ignoring the collisional de-excitation process, the ratio of the populations in the fine-structure levels of [O III] can be written as

$\frac{n_{2}}{n_{1}} \sim \frac{A_{1}}{A_{2}} \frac{q_{02}}{q_{01}+q_{02}}$,

where $n_{1}$ and $n_{2}$ represent the population numbers, $A_{1}$ and $A_{2}$ are the Einstein coefficients, and $q_{01}$ and $q_{02}$ are the rate coefficients for collisional excitation for ${ }^{3} P_{1}$ and ${ }^{3} P_{2}$ fine-structure levels, respectively. Then, the intensity ratio becomes:

$\frac{I_{52 \mu \mathrm{m}}}{I_{88 \mu \mathrm{m}}}=1.67 \frac{q_{02}}{q_{01}+q_{02}}$.

Lower limits of the $\left[\mathrm{O}_{\mathrm{III}}\right]$ line intensity ratio for $T_{\mathrm{e}}=$ $10000 \mathrm{~K}$ are derived as $0.563,0.475$, and 0.591 , for the collision strengths provided by Aggarwal et al. (1982), Bhatia et al. (1979), and Osterbrock (1974), respectively. The errors in the theoretical low density limit may be in the range $10-20 \%$, but they are unlikely to explain the data points below 0.4. If we take the coefficients by Bhatia et al. (1979), the mean electron density of the surrounding region becomes $90 \pm 50 \mathrm{~cm}^{-3}$. The derived upper limit of the density is not significantly affected by the errors of the collision coefficients in the range considered here.

According to the investigations based on the IRAS or COBE/DIRBE data, the effect of the interstellar extinction is negligible in this direction in FIR wavelengths (Sodroski 1994, 1997). However, the estimate is made under the assumption of the presence of a single temperature dust component along the line of sight. If there is a cold dust component, the optical depth $\tau_{100}$ may have been underestimated. The amount of the cold dust component on the line of sight may be able to be estimated from $\mathrm{CO}$ observations.

According to Drain \& Lee (1984), the extinction optical depth $\tau_{\text {ext }}$ can be given by

$\tau_{\text {ext }}=2.4 \times 10^{-24} \times N_{\mathrm{H}} \quad$ at $50 \mu \mathrm{m}$

and

$\tau_{\text {ext }}=6.5 \times 10^{-25} \times N_{\mathrm{H}} \quad$ at $100 \mu \mathrm{m}$,

where $N_{\mathrm{H}}$ is the column density of the hydrogen nuclei. It can be estimated by the observed $\operatorname{CO}(J=1-0)$ intensity as

$N_{\mathrm{H}}=2 N_{\mathrm{H}_{2}}=2 X W_{\mathrm{CO}}$, where $X$ is the conversion factor between the $\mathrm{H}_{2}$ column density and ${ }^{12} \mathrm{CO}(J=1-0)$ intensity, and is assumed to be $3 \times 10^{20} \mathrm{~mol} \mathrm{~cm}^{-2} \mathrm{~K}^{-1} \mathrm{~km}^{-1} \mathrm{~s}$ (Sodroski et al. 1997), and $\mathrm{W}_{\mathrm{CO}}$ is the observed ${ }^{12} \mathrm{CO}(J=1-0)$ intensity integrated over the velocity along the line of sight. From Eqs. (4)-(6) we have $\tau_{\text {ext }}(50 \mu \mathrm{m}) \sim 4 \tau_{\text {ext }}(100 \mu \mathrm{m}) \sim 1.5 \times 10^{-3} W_{\mathrm{CO}}$.

The high-resolution ${ }^{12} \mathrm{CO}(J=1-0)$ observations by Brooks et al. (1998) indicate that the molecular gas in the Carina region has clumpy morphology and the maximum integrated intensity seen is $200 \mathrm{~K} \mathrm{~km} \mathrm{~s}^{-1}$ at $(l, b)=\left(287^{\circ} 37,-0.63\right)$. With $W_{\mathrm{CO}}=200 \mathrm{~K} \mathrm{~km} \mathrm{~s}^{-1}$ and $\tau_{\text {ext }}(50 \mu \mathrm{m})=0.3$ the line ratio would be decreased by $25 \%$ if the line emitting gas is located behind the molecular gas. Outside of this peak, however, the integrated intensity is mostly less than $100 \mathrm{~K} \mathrm{~km} \mathrm{~s}^{-1}$. The low-resolution map by the Columbia survey (Grabelsky et al. 1988) does not show any other intensity clumps. Particularly the integrated intensity in the optically bright region and the surrounding region is much less than $20 \mathrm{~K} \mathrm{~km} \mathrm{~s}^{-1}$, indicating that the extinction should be negligible at $50 \mu \mathrm{m}$. The conversion factor $X$ varies with the environments and/or the galactocentric distance (e.g., Arimoto et al. 1996; Sodroski et al. 1997). However, the variation in our Galaxy is $\pm 30 \%$, and it does not affect the conclusion. At the positions where a large CO clump was observed (Brooks et al. 1998), the line ratios are always lower than 0.4. The optical depth at $50 \mu \mathrm{m}$ could be large there and we can attribute the low ratio to the dust extinction. There are a few other positions where a low line ratio was observed. No CO clumps have been reported toward these positions. We cannot rule out the possibility that some clumpy high-density gas intervenes the emission in these particular lines of sight. We do not have clear explanations for the points of significantly low line ratio $(<0.3)$. They all correspond to the positions of very faint [O III] emission and the measurement errors may partly be responsible for the low ratio. In general, the extinction should not make significant effects on the derived electron density for most of the observed positions based on the FIR and CO observations. Therefore, the low electron density derived in the molecular and surrounding regions is rather a secure conclusion.

\section{Discussion}

\subsection{Distribution of the ionized gas}

Two distinct components can be seen in the electron density map (Fig. 8). One is the relatively high density region enclosing Car I and Car II. We call this component "core ionized region". It corresponds to the optically bright region or the region of strong radio emission (Gardner et al. 1970). The electron density of this region is in the range $100-350 \mathrm{~cm}^{-3}$ except for two positions with $n_{\mathrm{e}} \geq 400 \mathrm{~cm}^{-3}$; one is the closest to $\eta$ Car, and the other is next east to Car I. At the $\eta$ Car position, we observed $\eta$ Car in the LWS $80^{\prime \prime}$ beam and the density should be affected by the gas surrounding $\eta$ Car. 
Observations of optical forbidden lines indicate $n_{\mathrm{e}}=$ $250-500 \mathrm{~cm}^{-3}$ in the nebula around $\eta$ Car (Peimbert et al. 1978), while the electron densities towards the Carina nebula have been derived to be $50-320 \mathrm{~cm}^{-3}$ from Si II $\lambda 1304.4$ observations (Walborn et al. 1984), $30-100 \mathrm{~cm}^{-3}$ from C II $\lambda 1335.7$ and $\lambda 1334.5$ observations (Walborn \& Hesser 1982), and larger than $300 \mathrm{~cm}^{-3}$ from UV observations of C I fine-structure lines (Walborn et al. 1998). They are in fair agreement with the present results for the core region.

Previous far-infrared observations have shown that several HII regions, such as M17, W51, and NGC 7538, have electron densities of the order of $10^{2}-10^{4} \mathrm{~cm}^{-3}$ (Watson et al. 1981; Moorwood et al. 1980). The electron density of the optically bright region is in the lower end of the range, suggesting that the Carina nebula is an evolved diffuse $\mathrm{H}$ II region.

Using the derived electron density, the extension of the ionized gas along the line of sight can be estimated. If we assume that the line is optically thin, the observed line intensity is given by

$I_{\mathrm{obs}}=\frac{h \nu}{4 \pi} A f\left(n_{\mathrm{e}}\right) n_{\mathrm{e}} \chi L$,

where $L$ is the path length along the line of sight, $A$ is the Einstein coefficient, $f\left(n_{\mathrm{e}}\right)$ is the fractional population of the ion excited in the upper level, and $\chi$ is the total elemental abundance of the emitting species relative to the hydrogen nuclei. The fractional population $f\left(n_{\mathrm{e}}\right)$ is a function of $n_{\mathrm{e}}$ and determined by the balance of the collisional excitation, de-excitation, and the spontaneous decay (Osterbrock 1989). For the [O III] $88 \mu \mathrm{m}$ transition it is approximately proportional to $n_{\mathrm{e}}$ for $n_{\mathrm{e}}<1000 \mathrm{~cm}^{-3}$. If we assume the abundance of oxygen relative to hydrogen $\left(\chi=6.6 \times 10^{-4}\right)$, ignoring the depletion in the highly ionized gas, and take the mean intensity of $6.3 \times 10^{-6} \mathrm{~W} \mathrm{~m}^{-2} \mathrm{sr}^{-1}$ for the [O III] $88 \mu \mathrm{m}$ line in the optically bright region, the corresponding gas extension on the line of sight $L$ becomes $0.4-4 \mathrm{pc}$ for $n_{\mathrm{e}}=100$ $350 \mathrm{~cm}^{-3}$, taking account of the absolute calibration uncertainty of $30 \%$. If we take the abundance determined by optical observations (Peimbert et al. 1978), $L$ becomes by $40 \%$ larger. The line-of-sight extension is slightly smaller than the spatial distance between the positions of $\eta \mathrm{Car}$ and the center of the Car I ( $\sim 10 \mathrm{pc})$. Thus the core ionized gas component is associated with Car I and Car II.

The other component seen in Fig. 8 is an extended low-density (ELD) ionized gas distributed all over the observed area. As discussed in the previous section, neither the possible calibration errors, the uncertainties in the collision coefficients, nor the extinction affects the low value of the derived electron density. The present results clearly indicate the presence of highly-ionized lowdensity $\left(n_{\mathrm{e}}<100 \mathrm{~cm}^{-3}\right)$ gas extending around the Carina $\mathrm{H}$ II region.

The mean electron density of the surrounding region is about $50 \mathrm{~cm}^{-3}$. If we take the mean intensity of the surrounding region of $4.5 \times 10^{-7} \mathrm{~W} \mathrm{~m}^{-2} \mathrm{sr}^{-1}$ as a representative value, the typical path length $L$ becomes $0.8 \mathrm{pc}$. The extended ionized gas is spread over the entire observed area, which corresponds to $30 \mathrm{pc}$ at the distance of the Carina nebula, indicating that the ionized gas is distributed in very thin sheets or filamentary structures. The filamentary nature of the ionized gas in the Carina region has also been suggested by optical observations (Smith et al. 2000). At 6 positions with the electron density of less than $20 \mathrm{~cm}^{-3}$, the path length $L$ becomes larger than $3 \mathrm{pc}$, suggesting a possibility that lower-density $\left(n_{\mathrm{e}}<20 \mathrm{~cm}^{-3}\right)$ ionized gas is distributed over the observed area superposed on the filamentary structures. The detection of the [O III] line at $b>0^{\circ}$ (Fig. 5a) also suggests that the diffuse $\mathrm{O}^{2+}$ gas is distributed in a scale larger than $50 \mathrm{pc}$.

Radio observations at $0.834 \mathrm{GHz}$ by Whiteoak (1994) show that the continuum intensity is approximately $3 \times$ $10^{-19} \mathrm{~W} \mathrm{~m}^{-2} \mathrm{~Hz}^{-1} \mathrm{sr}^{-1}$ and $2 \times 10^{-20} \mathrm{~W} \mathrm{~m}^{-2} \mathrm{~Hz}^{-1} \mathrm{sr}^{-1}$ for the optically bright region and the surrounding region, respectively. The free-free emission from the $[\mathrm{O}$ III] line emitting gas is estimated to be approximately $1 \times 10^{-19}$ and $1 \times 10^{-20} \mathrm{~W} \mathrm{~m}^{-2} \mathrm{~Hz}^{-1} \mathrm{sr}^{-1}$, respectively, according to Spitzer (1978) and thus contributes to $30-50 \%$ of the observed radio continuum. There should be a non-negligible amount of gas of lower ionization degree on the line of sight. The present results indicate that highly-ionized gas containing $\mathrm{O}^{2+}$ contributes to a significant fraction of the radio continuum in the Carina region.

There are several positions with large electron density $\left(n_{\mathrm{e}}>100 \mathrm{~cm}^{-3}\right)$ in the molecular cloud direction and in the surrounding region indicated by the present study. These may be attributed to density clumps. The positions of relatively high electron density in the molecular and surrounding regions are denoted as clump in the last column of Table 1. Further observations are needed to elucidate the physical nature of these density clumps.

The $[\mathrm{NII}] 122 \mu \mathrm{m}$ line was detected at most of the observed positions as well as [O III] and [N III] lines. It should be emitted in different physical conditions of lower density regions than $[\mathrm{O} \mathrm{III}]$ and $[\mathrm{N} \mathrm{III]}$ lines. There should be overlapping components of various density or ionization degree on the line of sight (cf., Walborn et al. 1998).

\subsection{ELD H II region}

The present observations indicate the presence of lowdensity ionized gas around the Carina nebula, whose electron density is less than $100 \mathrm{~cm}^{-3}$. The extended low density (ELD) ionized region was first investigated in detail by Mezger (1978) to account for the extended radio continuum emission from the Galactic plane. He suggested that the ELD HII regions are created by overlapping of evolved and expanded photoionized regions surrounding early-type stars and estimated the electron density as about $3 \mathrm{~cm}^{-3}$. The physical parameters of the ELD H II region were, however, not well determined because of the difficulty in observations of the diffuse component. Recently the diffuse warm ionized medium (WIM) or the 
"Reynolds Layer" has been discovered in our Galaxy by optical emission line studies (Reynolds 1990; Kulkarni \& Heiles 1988). It has been shown that a large fraction of the Galaxy contains fully ionized gas of $8000 \mathrm{~K}$ with an electron density of approximately $0.2 \mathrm{~cm}^{-3}$. Low ionization lines, such as [S II] $\lambda 6716$ and $[\mathrm{N} \mathrm{II}] \lambda 6587$, have been widely detected in the WIM and they have been used for the study of the properties of the WIM (e.g., Haffner et al. 1999 and references therein), while a high ionization line of [O III] $\lambda 5007$ is quite weak and has been detected only at two positions in the Galactic disk (Reynolds 1985). Latest studies suggest the presence of external heat sources in the Galactic halo to explain the spatial distribution and strength of these emission lines (Reynolds 1997; Reynolds et al. 1999). The Galactic disk, however, may be opaque to the optical lines and thus FIR lines are a useful means to study the diffuse ionized gas in the disk over the Galactic scale. COBE/FIRAS observations have detected low-ionized diffuse [N II] $122 \mu \mathrm{m}$ and $205 \mu \mathrm{m}$ lines and suggested that they come from the low-density diffuse ionized gas (Wright et al. 1991; Bennet et al. 1994). The present observations detected the FIR lines of highlyionized ions in the diffuse gas in the Galactic disk. The electron density $\left(n_{\mathrm{e}} \sim 50 \mathrm{~cm}^{-3}\right)$ derived in the present observations is significantly large compared to those estimated for the WIM or Reynolds Layer, suggesting that the detected highly-ionized diffuse gas may be a new class of the ISM components.

The WIM or the diffuse ionized gas (DIG) has been detected by optical observations also in external galaxies (for reviews, Dettmar 1992; Walterbos \& Braun 1996). In some galaxies a strong [O III] $\lambda 5007$ line has been observed in contrast to our Galaxy (Wang et al. 1997; Martin 1997; Greenawalt et al. 1997; Rand 1998; Hoopes et al. 1999). The ionization and heating source for the DIG in galaxies is still an open question, but the DIG should play an important role in the energy balance in the ISM in galaxies (e.g., Hoopes et al. 1999; Otte \& Dettmar 1999). Recently, Braine \& Hughes (1999) presented a spectrum of a normal galaxy, NGC 4414, for a wide spectral range. They detected [O III] $88 \mu \mathrm{m}$ line in their LWS spectrum and attributed it to classical $\mathrm{H}$ II regions because no Galactic [O III] emission had been measured, while [N II] $122 \mu \mathrm{m}$ line was suggested to come from the extended low-density ionized medium. Based on the [C II] $158 \mu \mathrm{m}$ and [N II] $122 \mu \mathrm{m}$ line intensities observed in 34 nearby galaxies, Negishi et al. (2001) have suggested that the average electron density of the [N II] emitting gas is about $35 \mathrm{~cm}^{-3}$ for their sample galaxies. The present observations, on the other hand, suggest that $[\mathrm{O} \mathrm{III}] 52$ and $88 \mu \mathrm{m}$ lines come from the ionized gas of slightly higher electron density spread over at least a tens-parsec scale in the Galactic plane.

The contribution of the ELD H II region to the energy of the Galactic diffuse radiation may be significant in the diffuse interstellar medium. Shibai et al. (1991, 1996) and Nakagawa et al. (1998) have detected diffuse [C II] $158 \mu \mathrm{m}$ emission on the Galactic plane by balloon-borne infrared telescopes and the Infrared Telescope in Space (IRTS), suggesting that the ELD H II region is one of the candidates for the source of the diffuse emission. Heiles (1994) indicated that the ELD H II region is the most likely candidate for the source of the diffuse [C II] emission. The ELD $\mathrm{H}$ II region they consider has a much smaller electron density $\left(n_{\mathrm{e}}<10 \mathrm{~cm}^{-3}\right)$ than the $\mathrm{O}^{2+}$ gas in the present study. COBE/FIRAS observations indicated that [C II $] 158 \mu \mathrm{m}$ emission is by 7 times stronger than [N II] $122 \mu \mathrm{m}$ emission and a dominant cooling line in the ISM. The present observations have shown that the intensity ratios relative to [N II] $122 \mu \mathrm{m}$ are 7, 18, and 29 for [N III] $57 \mu \mathrm{m}$, [O III] $52 \mu \mathrm{m}$, and $88 \mu \mathrm{m}$, respectively, suggesting that these ionic lines from the higher-density gas $\left(n_{\mathrm{e}} \sim 50 \mathrm{~cm}^{-3}\right)$ are dominant coolants in the Carina region compared to the general ISM. With $n_{\mathrm{e}}=50 \mathrm{~cm}^{-3}$ we derive the average column mass of the $\mathrm{O}^{2+}$ gas as $3 \times 10^{-6} \mathrm{~g} \mathrm{~cm}^{-2}$ for the surrounding region. The column number density of neutral hydrogen is estimated to be $1.4 \times 10^{22} \mathrm{~cm}^{-2}$ in the direction toward the Carina nebula based on $\mathrm{H}$ I $21 \mathrm{~cm}$ observations (Dickey \& Lockman 1990). The diffuse [OIII] emitting gas represents about $1 \%$ in mass of the neutral component in the Carina nebula direction.

\subsection{Heating sources}

In the observed region, there are many young, massive stars, which are members of the Tr 14 and 16 clusters and are considered to be the main heating sources. Otype stars in this region have been compiled by Walborn (1973). We investigate whether the ionizing photon in the region is supplied by these O-type stars. Hydrogen and helium atoms affect the ionizing radiation field and thus the calculation of photoionization of $\mathrm{O}^{+}$is not straightforward. We simply estimate the number of photons required to ionize hydrogen atoms in the nebula, in which all $\mathrm{O}^{+}$is assumed to exist. The Lyman-continuum (LyC) luminosity from O-type stars has been calculated by Vacca et al. (1996). The total LyC photon luminosity from five O3Vtype stars and one each of O3I, O5V, O5III, O6V, O6III, $\mathrm{O} 6.5 \mathrm{~V}$, and $\mathrm{O} 7 \mathrm{~V}$-type stars is calculated as $6.9 \times 10^{50} \mathrm{~s}^{-1}$ if the absorption is neglected. The number of LyC photons required to produce the $\mathrm{O}^{2+}$ region at each observed position, $N_{\text {LyC }}$, may be estimated as

$N_{\mathrm{LyC}}=\alpha n_{\mathrm{e}} n_{\mathrm{p}} L \Sigma D^{2}$,

where $\alpha$ is the recombination coefficient of hydrogen (Spitzer 1978), $n_{\mathrm{e}}$ and $n_{\mathrm{p}}$ are the densities of electron and proton, and $\Sigma$ and $D$ are the angular size of the area and the distance $(=2.7 \mathrm{kpc})$, respectively. We assume that the observed line intensities represent those of the corresponding grid area and set $\Sigma=3^{\prime} \times 3^{\prime}$. The density $n_{\mathrm{p}}$ is assumed to be equal to $n_{\mathrm{e}}$. We adopt the recombination coefficient of $2.6 \times 10^{-13} \mathrm{~cm}^{3} \mathrm{~s}^{-1}$ for case B of $T_{\mathrm{e}}=10000 \mathrm{~K}$ from Osterbrock (1989). The total number of $\mathrm{LyC}$ for the entire observed region is then estimated to be $5.4 \times 10^{49} \mathrm{~s}^{-1}$. This is a lower limit because the extinction is neglected and because the hydrogen ionized region is more extended than $\mathrm{O}^{2+}$ gas. However it seems unlikely that the estimate 
is too low by more than a factor of 10 . The O-stars in the Carina nebula seem to supply a sufficient number of $\mathrm{LyC}$ photons to produce the observed $\mathrm{O}^{2+}$ gas and external ionizing sources are not strongly called for.

\subsection{Ionic abundance}

The ratio of [N III] $57 \mu \mathrm{m}$ to [O III $52 \mu \mathrm{m}$ can be derived by a similar calculation as Eq. (1), assuming that both ions exist in the same region of the constant temperature and density. The calculation shows that the ratio of [N III] $57 \mu \mathrm{m}$ to [O III $52 \mu \mathrm{m}$ is almost constant as long as the electron density is less than $100 \mathrm{~cm}^{-3}$ and starts to decline for $n_{\mathrm{e}} \geq 100 \mathrm{~cm}^{-3}$. Assuming that $\mathrm{O}$ and $\mathrm{N}$ are in the solar abundance and both are completely in the stage of $\mathrm{O}^{2+}$ and $\mathrm{N}^{2+}$ ions, the line ratio at the low density limit of $T_{\mathrm{e}}=7500 \mathrm{~K}$ is 0.34 and becomes 0.25 at $n_{\mathrm{e}}=200 \mathrm{~cm}^{-3}$. The latter should be compared with the observed intensity ratio 0.27 of the optically bright region. For the surrounding region the observed ratio ranges $0.25-0.5$. Thus the line ratio of [N III] $57 \mu \mathrm{m}$ to [O III] $52 \mu \mathrm{m}$ also supports the low electron density of the emitting gas. In the optically thin case, the observed line ratio indicates that the abundance ratio of $\mathrm{N}^{2+} / \mathrm{O}^{2+}$ is approximately 0.19 in the core ionized gas. This is in agreement with those obtained in compact H II regions at the galactocentric distance of $8 \mathrm{kpc}$ (Roelfsema et al. 1998). The extended component is also suggested to have a similar $\mathrm{N}^{2+} / \mathrm{O}^{2+}$ abundance ratio to that in compact $\mathrm{H}$ II regions at a similar galactocentric distance.

The good correlation between [O III] and [N III] (Fig. 4) suggests that this ratio indeed indicates the ion abundance directly. $\mathrm{O}^{+}$has a slightly higher ionization potential than that of $\mathrm{N}^{+}$. Simpson et al. (1995) and Stasińska \& Schaerer (1997) have shown that the ratio of $n\left(\mathrm{O}^{2+}\right) / n(\mathrm{O})$ to $n\left(\mathrm{~N}^{2+}\right) / n(\mathrm{~N})$ is unity if the excitation temperature $T_{\mathrm{ex}}$ of the source is sufficiently high and it decreases with decreasing $T_{\text {ex }}$. Taking account of the fact that oxygen is more depleted than nitrogen in the ISM (Savage \& Sembach 1996) and assuming the solar abundance, the observed ratio of $n\left(\mathrm{O}^{2+}\right) / n\left(\mathrm{~N}^{2+}\right)$ to $[n(\mathrm{O}) / n(\mathrm{~N})]_{\odot}$ gives a lower limit of the true ionization fraction. According to Simpson et al. (1995) and Stasińska \& Schaerer (1997), the observed ratio of $0.25-0.5$ suggests that the excitation temperature of the ionizing source should be larger than $35000 \mathrm{~K}$ and thus the ionizing radiation must be quite hard. The O-type stars in the Carina star clusters provide sufficiently hard radiation. If external heating sources are required, they must ionize oxygen to the $\mathrm{O}^{2+}$ state efficiently.

\section{Summary}

We present the results of ISO/LWS observations of the diffuse interstellar medium around the star forming region, the Carina nebula. The observed region is an area of $40^{\prime} \times$ $20^{\prime}$ enclosing two large H II regions, Car I and II, which are heated by star clusters of Tr 14 and 16 .
[O III] $52,88 \mu \mathrm{m}$ and [N III] $57 \mu \mathrm{m}$ lines have been detected for almost all the observed positions. The present observations clearly show the existence of the extended highly-ionized low-density gas away from the optically bright region. The ratio of the [O III] lines indicates that the electron density is $100-350 \mathrm{~cm}^{-3}$ for the bright ionized region and less than $100 \mathrm{~cm}^{-3}$ for the surrounding region. The mean electron density $\left(\sim 50 \mathrm{~cm}^{-3}\right)$ and intensity of the latter component suggests a filamentary structure of the ionized gas. The electron density of this component is larger than those derived for the WIM or Reynolds layer and the ionized gas detected in the present study may be a new class of component of the ISM. The present study shows the presence of highly-ionized low-density ionized gas directly by far-infrared spectroscopy in the Galactic disk for the first time. The results demonstrate the importance of far-infrared observations from cooled telescopes for the study of diffuse ISM. Further investigations including other lines are warranted to elucidate the nature of the diffuse ISM.

Acknowledgements. We would like to thank the anonymous referee for several helpful suggestions, which improved the manuscript. We also thank the LWS IDT in Rutherford Appleton Laboratory, particularly S. Sidher, for their help in the LWS data processing, M. Burgdorf for the latest information on the beam size of LWS, and K. Kawara, Y. Satoh, H. Okuda, and the Japanese ISO team for their continuous help and encouragement. This work was supported in part by Grant-in-Aids for Scientific Research from Japanese Society for the Promotion of Science.

\section{References}

Aggarwal, K. M., Baluja, K. L., \& Tully, J. A. 1982, MNRAS, 201, 923

Arimoto, N., Sofue, Y., \& Tsujimoto, T. 1996, PASJ, 48, 275

Bennett, C. L., Fixsen, D. J., Hinshaw, G., et al. 1994, ApJ, 434,587

Bhatia, A. K., Doschek, G. A., \& Feldman, U. 1979, A\&A, 76, 359

Braine, J., \& Hughes, D. H. 1999, A\&A, 344, 779

Brooks, K. J., Whiteoak, J. B., \& Storey, J. W. V. 1998, PASA, 15, 202

Burgdorf, M., Clegg, P., Ewart, D., et al. 1997, in First ISO Workshop on Analytical Spectroscopy, ed. A. M. Heras, K. Leech, N. R. Trams, \& M. Perry, ESA SP-419, 51

Clegg, P. E., Ade, P. A. R., Armand, C., et al. 1996, A\&A, 315, L38

Cox, P., \& Bronfman, L. 1995, A\&A, 299, 583

de Graauw, T., Lidholm, S., Fitton, B., et al. 1981, A\&A, 102, 257

Deharveng, L., \& Maucherat, M. 1975, A\&A, 41, 27

Dettmar, R.-J. 1992, Fundam. Cosmic Phys., 15, 143

Dickel, H. R. 1974, A\&A, 31, 11

Dickey, J. M., \& Lockman, F. J. 1990, ARA\&A, 28, 215

Drain, B. T., \& Lee, H. M. 1984, ApJ, 285, 89

Feinstein, A. 1995, Rev. Mex. Astron. Astrofis., 2, 57

Gardner, F. F., \& Morimoto, M. 1968, Aust. J. Phys., 21, 881

Gardner, F. F., Milne, D. K., Mezger, P. G., \& Wilson, T. L. 1970, A\&A, 7, 349 
Ghosh, S. K., Iyengar, K. V. K., Rengarajan, T. N., et al. 1988, ApJ, 330, 928

Grabelsky, D. A., Cohen, R. S., Bronfman, J., \& Thaddeus, P. 1988, ApJ, 331, 181

Greenawalt, B., Walterbos, R. A. M., \& Braun, R. 1997, ApJ, 483, 666

Gry, C. 2000, The ISO Handbook volume IV: LWS - The Long Wavelength Spectrometer, Ver. 1.0

Haffner, L. M., Reynolds, R. J., \& Tufte, S. L. 1999, ApJ, 523, 223

Harvey, P. M., Hoffman, W. F., \& Campbell, M. F. 1979, ApJ, 227, 114

Heiles, C. 1994, ApJ, 436, 720

Hoopes, C. G., Walterbos, R. A. M., \& Rand, R. J. 1999, ApJ, 522, 669

Kessler, M. F., Steinz, J. A., Anderegg, M. E., et al. 1996, A\&A, 315, L27

Kulkarni, S. R., \& Heiles, C. 1988, in Galactic and Extragalactic Radio Astronomy, 2d ed., ed. G. L. Verschuur, \& K. I. Kellermann (Springer, Berlin and New York), 95

Martin, C. L. 1997, ApJ, 491, 561

Mezger, P. G. 1978, A\&A, 70, 565

Moorwood, A. F. M., Baluteau, J.-P., Anderegg, M., et al. 1980, ApJ, 238, 565

Nakagawa, T., Yui, Y. Y., Doi, Y., et al. 1998, ApJS, 115, 259

Negishi, T., Onaka, T., Chan, K.-W., \& Roellig, T. L. 2001, A\&A, 375, 566

Osterbrock, D. E. 1974, Astrophysics of Gaseous Nebulae (Freeman, San Francisco)

Osterbrock, D. E. 1989, Astrophysics of Gaseous Nebulae and Active Galactic Nuclei (University Science Books, Mill Valley)

Otte, B., \& Dettmar, R. J. 1999, A\&A, 343, 705

Peimbert, M., Torres-Peimbert, S., \& Rayo, J. F. 1978, ApJ, 220,516

Rand, R. J. 1998, ApJ, 501, 137

Retallack, D. S. 1983, MNRAS, 204, 669

Reynolds, R. J. 1985, ApJ, 298, L27

Reynolds, R. J. 1990, in The Galactic and Extragalactic Background Radiations, ed. S. Bowyer, \& C. Leinert, IAU Symp., 139, 157

Reynolds, R. J. 1997, Nature, 277, 1446
Reynolds, R. J., Haffner, L. M., \& Tufte, S. L. 1999, ApJ, 525, L21

Roelfsema, P. R., Cox, P., Kessler, M. F., \& Baluteau, J.-P. 1998, in Star Formation with the Infrared Space Observatory (ISO), ed. J. L. Yun, \& R. Liseau, ASP Conf. Ser., 132, 76

Savage, B. D., \& Sembach, K. R. 1996, ARA\&A, 34, 279

Shibai, H., Okuda, H., Nakagawa, T., et al. 1991, ApJ, 374, 522

Shibai, H., Okuda, H., Nakagawa, T., et al. 1996, PASJ, 48, L127

Simpson, J. P. 1975, A\&A, 39, 43

Simpson, J. P., Colgan, S. W. J., Rubin, R. H., et al. 1995, ApJ, 444, 721

Smith, N., Egan, M. P., Carey, S., et al. 2000, ApJ, 532, L145

Sodroski, T. J., Bennett, C., Boggess, N., et al. 1994, ApJ, 428, 638

Sodroski, T. J., Odegard, N., Arendt, R. G., et al. 1997, ApJ, 480, 173

Spitzer, L., Jr. 1978, Physical Processes in the Interstellar Medium (Wiley, New York)

Stasińska, G., \& Schaerer, D. 1997, A\&A, 322, 615

Vacca, W. D., Garmany, C. D., \& Shull, J. M. 1996, ApJ, 460, 914

Walborn, N. R. 1973, ApJ, 179, 517

Walborn, N. R. 1995, Rev. Mex., A\&A, 2, 51

Walborn, N. R., \& Hesser, J. E. 1982, ApJ, 252, 156

Walborn, N. R., Danks, A. C., Sembach, K. R., et al. 1998, ApJ, 492, L169

Walborn, N. R., Heckathorn, J. M., \& Hesser, J. E. 1984, ApJ, 276, 524

Walterbos, R. A., \& Braun, R. 1996, in The Minnesota Lectures on Extragalactic Neutral Hydrogen, ed. E. D. Skillman, ASP Conf. Ser., 106, 1

Wang, J., Heckman, T. M., \& Lehnert, M. D. 1997, ApJ, 491, 114

Watson, D. M., Storey, J. W. V., Townes, C. H., \& Haller, E. E. 1981, ApJ, 250, 605

Whiteoak, J. B. Z. 1994, ApJ, 429, 225

Whiteoak, J. B., \& Otrupcek, R. E. 1984, PASA, 5, 552

Wright, E. L., Mather, J. C., Bennett, C. L., et al., 1991, ApJ, 381,200 